\title{
Modelando dinámicas de movilidad y visibilidad en los paisajes megalíticos gallegos. El caso del Monte de Santa Mariña y su entorno (Comarca de Sarria, Lugo)
}

\author{
Modelling mobility and visibility dynamics in Galician megalithic moundscapes. \\ The case of Monte de Santa Mariña and its surroundings (Sarria's region, Lugo)
}

\author{
Miguel Carrero-Pazos ${ }^{\mathrm{a}}$
}

\author{
A Antón A. Rodríguez Casal y Marcos Mar- \\ tinón-Torres.
}

\section{RESUMEN}

Se propone un estudio de dos formas tradicionales de entender la relación del megalito con el paisaje, como son la movilidad natural por el territorio y la visibilidad del monumento con respecto al paisaje en el que se emplaza. Para ello, se aplican una serie de análisis fundamentados en los Sistemas de Información Geográfica y la Estadística Espacial sobre el conjunto tumular del Monte de Santa Mariña (Comarca de Sarria, Lugo) y su entorno (NO de la Península Ibérica). Partiendo de la creación de un modelo de simulación de desplazamiento natural y del estudio de la visibilidad de dicho conjunto, se comprueba, en primer lugar, la importancia de las vías potenciales de tránsito como variable locacional, así como el impacto visual que los monumentos juegan en relación con estas vías. A continuación, se analiza la prominencia visual de los túmulos megalíticos con respecto a su entorno inmediato, pudiéndose identificar la existencia de tendencias comunes en los distintos conjuntos tumulares en relación con el tamaño de las cuencas visuales. Los resultados sugieren una importante relación entre los túmulos y el tránsito natural por el territorio, si bien se detectan tendencias desiguales en lo que al impacto visual del megalito se refiere, tanto con respecto a la relación con otros túmulos como a la totalidad del territorio en el que se emplazan. Ello permite establecer un punto de partida necesario para la revisión de los planteamientos teóricos formulados para el conjunto de Galicia.

\begin{abstract}
This paper aims to analyse two traditional forms of association of the megaliths with the landscape, such as the natural mobility and the visibility of the landscape. Several analytical approaches based on Geographic Information Systems and Spatial Statistics are applied over the megalithic barrow landscape of Monte de Santa Mariña (Sarria's region, Lugo) and its surroundings (NW of the Iberian Peninsula). Starting from the creation of a simulation model of natural movement and the analysis of the visibility of sites, the relevance of the potential transit routes as a locational variable is checked, as well as the role that visibility of these monuments may have played regarding these routes. Subsequently, the visual prominence of the monuments with background terrain is also analysed, being able to observe the existence of common tendencies in different necropoleis of this megalithic complex concerning the size of the visual basin. The results allow us to consider the existence of an important relation between the megalithic monuments and the natural passage through the territory, with unequal trends on the visual impact of the megalith, regarding the relation with other sites and the whole territory in which the necropolis is located, which seems to indicate the necessity to reconsider the traditional discourse using these regional trends.
\end{abstract}

Palabras clave: Megalitismo; SIG; Análisis espacial; Patrones de localización; Caminos óptimos; Visibilidades; Simulación de Monte Carlo; $R$ Statistics; GRASS GIS; Galicia; Neolítico.

Key words: Megalithic complex; GIS; Spatial Analysis; Locational patterns; Least Cost Path; visibility; Monte Carlo Simulation; R Statistics; GRASS GIS; Galicia; Neolithic.

a Universidade de Santiago de Compostela, Grupo de Estudos para a Prehistoria do Noroeste Ibérico-Arqueoloxía,Antigüidade e Territorio (GEPN-AAT). Facultade de Xeografía e Historia. Praza da Universidade 1. 15782 Santiago de Compostela. Correo e.: miguel.carrero.pazos@gmail.com https://orcid-org/0000-0001-9203-9954

Recibido 4-I-2018; aceptado 23-III-2018. 


\section{INTRODUCCIÓN}

El megalitismo constituye un fenómeno social y cultural común a toda la fachada atlántica europea, cuyos inicios se sitúan, en términos generales, hacia comienzos del V milenio a. C. Se caracteriza principalmente en el plano arquitectónico por la construcción de monumentos pétreos. Mientras que las intervenciones en el paisaje a pequeña escala definieron las sociedades preneolíticas y/o no agrarias, el período neolítico se caracterizó por un cambio en el grado en el que los seres humanos comprendieron, organizaron y transformaron dicho paisaje. Ello se produjo, entre otros elementos, a través de la construcción de unos monumentos funerarios que, perpetuándose en el tiempo, vertebraron los territorios de las sociedades vivas (Fleming 1973; García Sanjuán 2000, 2008; Martinón-Torres 2001).

Estos monumentos pueden entenderse como el resultado de la forma como las sociedades neolíticas entendieron y estructuraron el espacio (Criado Boado 1989; Bradley 1998). En consecuencia, si se consigue analizar y aprehender los criterios que determinaron la localización de tales monumentos, estaremos en disposición de aproximarnos a cómo dichas sociedades organizaron sus territorios. Una de las opciones metodológicas para el estudio de los patrones territoriales del pasado son los Sistemas de Información Geográfica (SIG), que permiten elaborar modelos para profundizar en el análisis del emplazamiento de los yacimientos (Parcero Oubiña y Fábrega Álvarez 2006; Llobera 2015).

El caso de estudio es el Monte de Santa Mariña y su entorno, un conjunto monumental del interior de Galicia con cuya investigación nos proponemos contribuir a una interpretación más objetiva y robusta de los patrones de localización tumulares, a partir de los propuestos por diferentes autores en otras áreas peninsulares y europeas (véanse p. ej., Cerrillo Cuenca 2007; De Reu 2012; Bourgeois 2013). En Galicia, el estudio de los patrones de localización tumulares puede dividirse conceptualmente en dos grupos. El primero comprende los trabajos derivados de una corriente espacial de índole clásica (véase, p. ej., Criado Boado y Grajal 1981; Bello Diéguez et al. 1982a, 1982b, 1987; Eguileta Franco 1999) y el segundo los surgidos de los planteamientos de la Arqueología del Paisaje (p. ej., Criado Boado, tesis inédita ${ }^{1}, 1989$, 1993; Vaquero Lastres 1990, 1993-1994; Criado Boado y Villoch Vázquez 1998; Villoch Vázquez, tesis

\footnotetext{
${ }^{1}$ Criado Boado, F. 1988: Contribución al estudio de las relaciones entre las comunidades megaliticas del noroeste peninsular y su medio natural: implicaciones socio-económicas. Tesis doctoral inédita. Universidade de Santiago de Compostela. Santiago de Compostela.
}

inédita ${ }^{2}$ ). En ambos, los autores trataron de identificar y caracterizar el patrón de ocupación megalítico analizando los factores diversos que pudieron determinar la localización de los monumentos megalíticos.

Desde finales de los 1980, los factores sugeridos con más frecuencia por investigadores como J. M. ${ }^{a}$ Bello Diéguez, F. Criado Boado o J. M. Vázquez Varela fueron la movilidad natural por el territorio y la visibilidad del monumento. Ambos factores habrían formado parte de las "especiales razones [que] determinaron la localización geográfica de los túmulos dolménicos tal y como hoy se conocen" (Criado Boado y Grajal 1981: 8).

Este trabajo toma como punto de partida la investigación de M. Llobera (2015), que constituye la primera aproximación a la visibilidad y movilidad en las necrópolis megalíticas gallegas a través de la aplicación de los SIG. En él se prioriza dos de los criterios de dicho autor - la movilidad natural por el territorio y la visibilidad del monumento con respecto al paisaje en el que se emplaza - para analizar y comprobar su importancia como variables locacionales del conjunto monumental del Monte de Santa Mariña y su entorno. Para ello, el estudio se apoya en la potencialidad analítica de los SIG y en la Estadística Espacial.

Sin lugar a dudas, F. Maciñeira $(1929,1935,1943-$ 1944), en sus trabajos ya clásicos, fue quien primero analizó en profundidad en Galicia la relación de los túmulos megalíticos — mámoas - con lugares de paso. Proponía, p. ej., que "muchos megalitos se levantaron en las márgenes de antiguas vías de comunicación", o que "as mámoas aliñábanse flanqueando os camiños existentes cando foron construidas" (Bello Diéguez et al. 1982a: 3, 1982b: 117). Apreciaciones de este tipo estaban ya presentes en las contribuciones de Á. Castillo López (1927), F. López Cuevillas $(1925,1933)$ o M. Díaz Sanjurjo (1904: 223), para quien la existencia de mámoas "es uno de los indicios más seguros en Galicia de la proximidad de los antiguos caminos". No obstante, fue en los 1980 cuando J. M. ${ }^{a}$ Bello Diéguez, F. Criado Boado y J. M. Vázquez Varela (1982a) publicaron el primer estudio exhaustivo de la importancia de este posible factor de localización. En documentación medieval identificaron caminos antiguos ("camiños reais"), observando numerosas mámoas en sus inmediaciones.

Esta masa crítica sentó las bases de una nueva línea de investigación, denominada "geografía de la movilidad o del desplazamiento" (Criado Boado y Vaquero Lastres 1991, 1993; Vaquero Lastres 1991-1992; Infante Roura et al. 1992; Eguileta Franco 1997, 1999). Entre

\footnotetext{
2 Villoch Vázquez, V. 2000: La configuración del espacio entre las sociedades constructoras de túmulos en Galicia: estudios de emplazamiento tumular. Tesis doctoral inédita. Universidade de Santiago de Compostela. Santiago de Compostela.
}

Trab. Prehist., 75, N. ${ }^{\circ}$ 2, julio-diciembre 2018, pp. 287-306, ISSN: 0082-5638

https://doi.org/10.3989/tp.2018.12216 
sus principales aportaciones destaca la vinculación entre vías de tránsito y megalitos como uno de los criterios locacionales de los mismos, "un factor de emplazamiento uniforme pues es inherente a la propia esencia de la construcción, su visibilidad" (Vaquero Lastres 19931994: 13). Las mámoas se relacionaban con las zonas de paso natural a partir de la identificación de elementos cartografiables como los caminos reales-tradicionales y las vías naturales de tránsito (Eguileta Franco 2000; Martinón-Torres 2001; Fábregas Valcarce 2010).

La visibilidad ha sido considerada como un factor de emplazamiento propio del fenómeno megalítico en un buen número de trabajos realizados en Galicia durante los 1990 (sin ánimo de ser exhaustivos, Criado Boado 1988, tesis inédita ${ }^{3}$, 1989b, 1993; Villoch Vázquez, tesis inédita ${ }^{4}$ ). Tal es su importancia que se le ha considerado el elemento fundamental para explicar la ubicación concreta de estos monumentos, un factor asociado a emplazamientos en zonas prominentes del terreno con un impacto visual sobre las parcelas cultivadas en la actualidad, que han sido interpretadas como posibles áreas de poblamiento durante el Neolítico (Criado Boado 1988 y tesis inédita ${ }^{5}$ ).

\section{2. ÁREA DE ESTUDIO}

La región seleccionada, situada al sur de la provincia de Lugo, alberga 75 monumentos megalíticos agrupados en torno a 6 conjuntos tumulares: Monte de Santa Mariña, O Edramo, Os Chaos, Bardaos, Chao de Nadelo y Monte Meda (Fig. 1; material complementario AC1). El del Monte de Santa Mariña es el que mayor atención ha recibido en la historiografía. Desde su primera cita en la "Carta Arqueológica del Ayuntamiento de O Incio" "la investigación ha subrayado el gran número de mámoas que contiene (Filgueiras Rey y Rodríguez Fernández 1994; Rodríguez Casal 1998; Rodríguez Casal et al. 1998; Carrero Pazos et al. 2014; Carrero Pazos y Rodríguez Casal 2015,7; Carrero Pazos y Vilas Estévez 2016).

La zona de trabajo se ubica en la comarca de Sarria, en los términos municipales de Láncara, Triacastela, Sarria, Samos y O Incio, en un entorno geográfico de llanuras, interfluvios, sierras y depresiones propias de

\footnotetext{
${ }^{3}$ Véase n. 1.

4 Véase n. 2.

${ }^{5}$ Véase n. 1.

${ }^{6}$ Memoria inédita, 1968

7 Carrero Pazos, M. y Rodríguez Casal, A. A. "General and local spatial trends in Galician megalithic landscapes". Edición previa de la comunicación presentada a la "Early monumentality and social differentiation in Neolithic Europe: megaliths, societies, landscapes conference (Kiel, Alemania 2015)". Prevista publicación en M. Hinz (ed.): Megaliths, societies and landscapes: early monumentality and social differentiation in Neolithic Europe. Universität zu Kiel, Kiel, 2017.
}

la Galicia centro-oriental. Se buscaron límites geográficos naturales en vez de administrativos, con el fin de acotar una zona más adecuada a las concentraciones monumentales, recurriendo para ello a la red fluvial ${ }^{8}$ y las cuencas hidrográficas potenciales. Dichas cuencas se estructuran en torno a las principales regiones montañosas de la parte central, caso del Monte de Santa Mariña y A Serra do Edramo, y de las estribaciones finales de las Sierras orientales gallegas como A Serra do Oribio, al sureste.

La delimitación fue modelizada en GRASS GIS en su versión 7.0.4 mediante la herramienta r.watershed ${ }^{9}$ que utiliza el algoritmo de cálculo multiple flow direction para crear un ráster de acumulación donde el flujo de agua se distribuye hacia todas las celdas vecinas con menor elevación, ponderando la distribución con el factor pendiente (Holmgren 1994) ${ }^{10}$. El cálculo fue repetido tomando como base diversos radios de cuenca hidrográfica. Se seleccionó el de $25 \mathrm{~km}$ al permitir la definición de un territorio cercano a las agrupaciones de monumentos (en torno a $10 \mathrm{~km}$ de buffer a la redonda) (Fig. 1).

La totalidad de la parte analítica se desarrolló con un Modelo Digital de Elevaciones (MDE) de $25 \mathrm{~m}$ de resolución, obtenido del Centro Nacional de Información Geográfica (CNIG). El uso de modelos digitales con mayor resolución - caso del MDE de $5 \mathrm{~m}$ derivado de datos Laser Imaging Detection and Ranging (LiDAR)aumentaría la precisión en los resultados. Sin embargo el elevado coste, computacionalmente hablando, de las operaciones analíticas, una circunstancia inviable en nuestra situación por el difícil acceso a la supercomputación $^{11}$, justifica la elección de un MDE de $25 \mathrm{~m}$.

\section{MODELIZANDO EL TRÁNSITO NATURAL CON LOS SIG}

Los estudios arqueológicos sobre tránsito relacionados con monumentos megalíticos se han centrado en su vinculación espacial con rutas de trashumancia, entendiendo éstas como posibles vestigios de caminos

\footnotetext{
${ }^{8}$ Cartografía en formato Shapefile, extraída de la Base Hidrográfica a escala 1:25.000 del Instituto Geográfico Nacional, con posterior modificación y actualización por parte del Instituto de Estudos do Territorio (IET, Xunta de Galicia).

9 GRASS Development Team 2016. Geographic Resources Analysis Support System (GRASS) Software, Version 7.0. Open Source Geospatial Foundation. Electronic document: https://grass.osgeo.org/grass $73 / \mathrm{manu}-$ als/r.watershed.html (consulta 20-08-2017).

${ }^{10}$ Esta herramienta incorpora además el algoritmo de búsqueda de menor coste $\mathrm{A}^{\mathrm{T}}$, diseñado para minimizar el impacto de los errores de los MDEs en el cálculo de los modelos hidrográficos (Ehlschlaeger 1989).

${ }^{11}$ Los cálculos fueron realizados sobre un ordenador portátil MSi Intel Core (TM) i7-4712 MQ CPU $2.30 \mathrm{GHz}$ con $16 \mathrm{~GB}$ de ram, trabajando sobre un sistema operativo Windows 10 .
} 

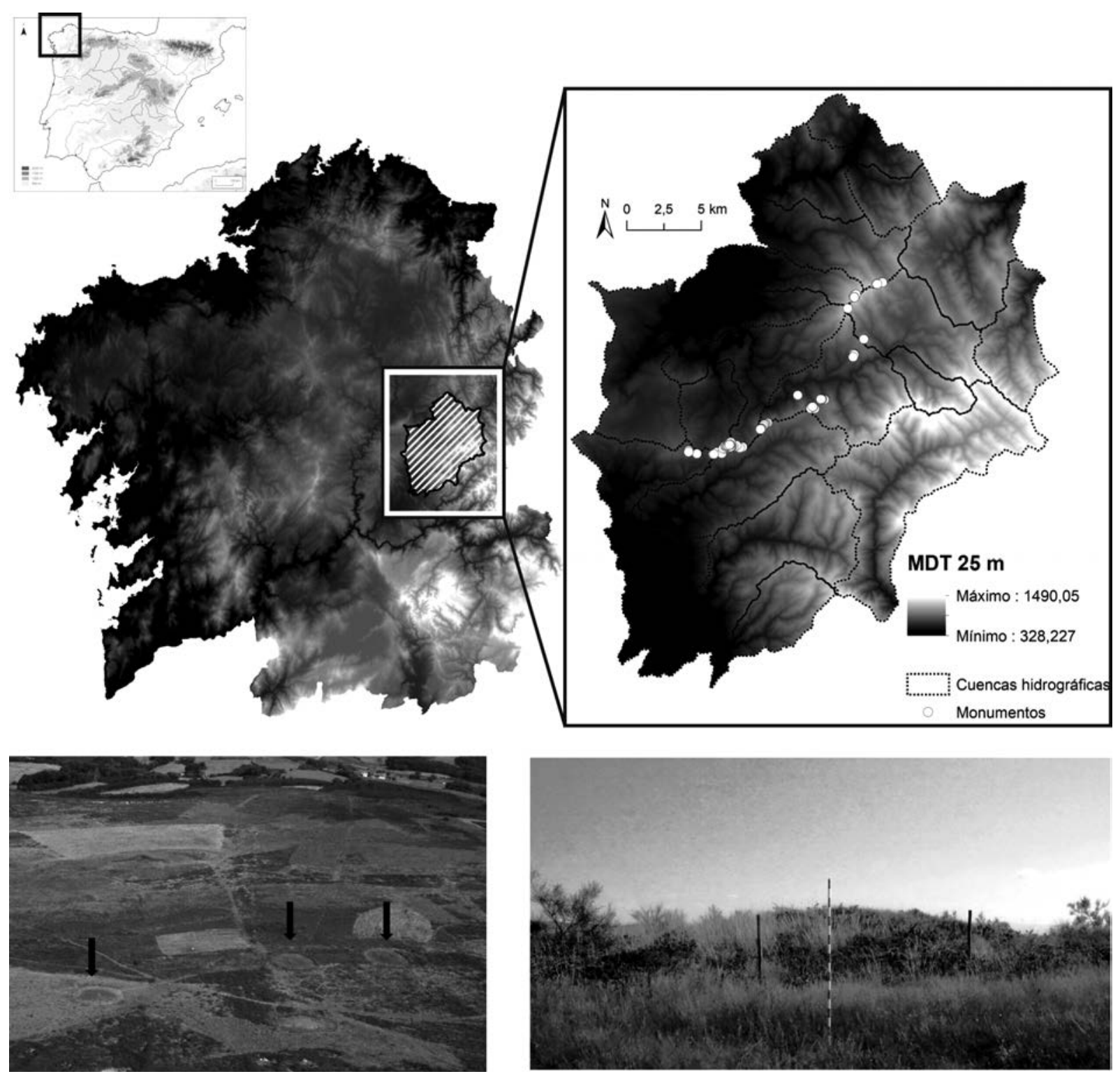

Fig. 1. Arriba: localización del área de estudio en la Península Ibérica y Galicia. Modelo digital de elevaciones de $25 \mathrm{~m}$ de resolución ( $\mathrm{m}$ s. n. m). Base cartográfica: Centro Nacional de Información Geográfica. Abajo: vista aérea del Monte de Santa Mariña (comarca de Sarria, Lugo), localizando tres túmulos megalíticos, y vista en superficie de uno de ellos (fotografías del Grupo de investigación-1520 "Arqueoloxía e ecoloxía do fenómeno tumular e megalítico galego". Vista aérea tomada en verano de 2005, y fotografía del túmulo en verano de 2015).

prehistóricos anteriores (Chapman 1979; Davidson 1980; Cara Barrionuevo y Rodríguez López 1987; Murrieta Flores 2010; Wheatley et al. 2010), así como en la consideración del megalito como marcador territorial (véanse, p. ej., Criado Boado et al. 1990-1991; Criado Boado y Vaquero Lastres 1993; Martinón-Torres 2001; López Plaza y Salvador Mateos 2002; Murrieta Flores et al. 2011a, 2011b). En el presente trabajo, sin embargo, la aproximación a la movilidad natural por el territorio - entendida como la estructura geográfica del movimiento por el paisaje- ni considera los caminos históricos como puntos clave del desplazamiento, ni tampoco los propios túmulos megalíticos como elementos articuladores de la movilidad.

Los modelos de movilidad humana desarrollados gracias a los SIG se concretan en modelos matemáticos de movimiento, aplicados a superficies ráster cuyas 
características determinan las condiciones del desplazamiento. El de la ruta de menor coste - o least cost path - es uno de los más utilizados. Se trata de la vía de tránsito generada entre dos puntos con el menor coste en tiempo o energía, en función de determinados factores (véase, p. ej., Llobera 2000; Bevan 2011; White y Barber 2012; Herzog 2013a, 2013b; Verhagen 2013; Mlekuz 2014; Murrieta Flores et al. 2014; Yubero-Gómez et al. 2015; Orengo y Livarda 2016).

Para la modelización con SIG de los corredores naturales de tránsito hemos optado por una metodología similar a la que M. Llobera (2015) empleó en el estudio del fenómeno tumular en la Península del Barbanza. En nuestro caso la representatividad analítica es menor tanto por el MDE utilizado como por el número de simulaciones efectuado. También utilizaron esa metodología P. Murrieta Flores et al. (2014) - aunque con particularidades-, en su estudio del megalitismo del sur peninsular, C. Rodríguez Rellán y R. Fábregas Valcarce (2015) en el que estudiaron el arte rupestre galaico, así como E. Cerrillo Cuenca (2007), P. Fábrega-Álvarez et al. (2011) o A. Bevan y A. Wilson (2013), entre otros.

Su aplicación en el Monte de Santa Mariña, con base en GRASS GIS - versión 7.0.4-, creó un modelo general de movilidad humana, a partir de la generación de múltiples rutas de menor coste que comienzan y finalizan en los límites del área de trabajo. Previamente esos límites fueron descompuestos en puntos cada $500 \mathrm{~m}$. El cálculo se realizó sobre un total de 458 puntos de partida y destino obtenidos. Para la creación de la superficie de coste acumulativo o representación del coste al tránsito se usaron las herramientas r.watershed y r.walk. Esta última generó una superficie ráster de coste de movimiento anisotrópico desde los límites del área de trabajo. Posteriormente, con r.drain se generaron los vectores de las rutas.

Este proceso fue repetido para la totalidad de los puntos de partida y destino, produciendo un total de 209.306 rutas. Finalmente, con la herramienta v.kernel, se creó un mapa ráster de concentración de rutas donde se destacan zonas con elevada densidad de rutas de menor coste. La tabla 1 resume el desarrollo metodológico. Los análisis realizados se concretan en una forma de movilidad que puede ser expresada desde un punto a la totalidad (one to many), si bien el cálculo se repite para un conjunto amplio de puntos (White y Barber 2012). Este método permite obtener múltiples rutas de tránsito que, sumadas, proporcionan corredores con mayor idoneidad para el desplazamiento. Ello los convierte en vectores clave o nodos teóricos en una red de tránsito peatonal (Rodríguez Rellán y Fábregas Valcarce 2015: 334) (Fig. 2A).

A partir de estos resultados podemos calcular con la herramienta r.walk el esfuerzo en tiempo (minutos) que tomaría llegar desde esos corredores al monumento megalítico, comprobando si hay o no una relación (y su intensidad) entre la localización de los túmulos y las rutas de tránsito potenciales (Fig. 2B).

El resultado de la intensidad de tránsito potencial obtenido para cada monumento en relación con el terreno sugiere una mayor presencia de monumentos en zonas con una elevada intensidad de tránsito, si bien existe un número elevado que se sitúa en zonas con un tránsito

\begin{tabular}{|c|c|c|c|}
\hline \multicolumn{5}{|c|}{ CÁLCULO DE DENSIDAD DE RUTAS ÓPTIMAS } \\
\hline Módulo utilizado & Objetivo & Input & Output \\
\hline v.to.points & $\begin{array}{c}\text { Descomponer el vector (línea) } \\
\text { del área de estudio en puntos } \\
\text { espaciados regularmente }(500 \mathrm{~m})\end{array}$ & $\begin{array}{c}\text { Vector de línea del área } \\
\text { de estudio }\end{array}$ & $\begin{array}{c}\text { Vector de línea del área de estudio } \\
\text { descompuesto en puntos regulares } \\
\text { cada 500 m }(n=498)\end{array}$ \\
\hline r.watershed & $\begin{array}{c}\text { Generar la superficie de } \\
\text { acumulación de flujo }\end{array}$ & $\begin{array}{c}\text { Modelo Digital } \\
\text { de Elevaciones }\end{array}$ & $\begin{array}{c}\text { Raster de acumulación } \\
\text { de agua potencial }\end{array}$ \\
\hline r.walk & $\begin{array}{c}\text { Generar la superficie de coste } \\
\text { de movimiento }\end{array}$ & $\begin{array}{c}\text { Origen: punto individual } \\
\text { Destino: vector de puntos }\end{array}$ & $\begin{array}{c}\text { Raster de coste de movimiento } \\
\text { anisotrópico }\end{array}$ \\
\hline r.drain & $\begin{array}{c}\text { Generar los vectores } \\
\text { de rutas óptimas }\end{array}$ & $\begin{array}{c}\text { Raster de coste de } \\
\text { movimiento anisotrópico } \\
\text { (origen: vector de puntos) }\end{array}$ & $\begin{array}{c}\text { Vector de rutas } \\
(n=209.306)\end{array}$ \\
\hline \multicolumn{7}{|c|}{ r.drain se repite $\boldsymbol{n}$ veces $(\boldsymbol{n}=\mathbf{4 5 8})$} & Rector de rutas & $\begin{array}{c}\text { Raster de densidad de rutas de } \\
\text { tránsito óptimas }\end{array}$ \\
\hline
\end{tabular}

Tab. 1. Descripción metodológica del cálculo de densidad de rutas óptimas, realizado con GRASS GIS en su versión 7.0.4 para la creación de un modelo general de movilidad humana en el Monte de Santa Mariña y su entorno. 
reducido. Asimismo, buena parte de los megalitos se sitúa en zonas próximas a las rutas de desplazamiento potencial expresado en tiempo (hasta 10 minutos, generalmente), una circunstancia que, en sintonía con los trabajos señalados más arriba, permite proponer la movilidad como posible factor locacional de primer orden.
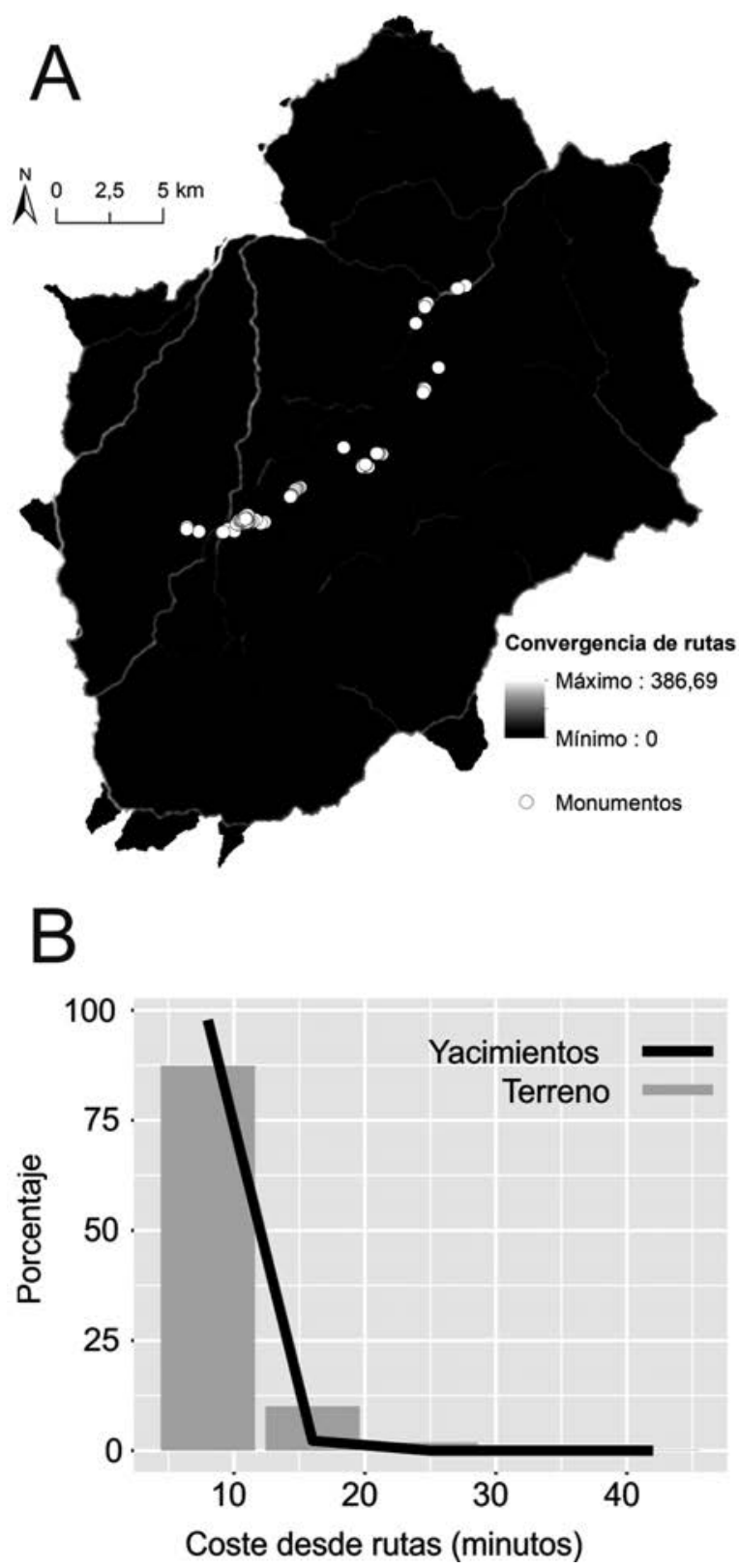

Fig. 2. Modelo general de movilidad humana en el Monte de Santa Mariña y su entorno. Base cartográfica: Centro Nacional de Información Geográfica, con una resolución de $25 \mathrm{~m}$. A. modelización de la intensidad de tránsito potencial; B. valores del coste de desplazamiento desde las rutas de tránsito potencial a los túmulos megalíticos.

\section{RECONSTRUYENDO LOS PAISAJES VISUALES}

La visibilidad es uno de los factores que, según la tradición historiográfica en Galicia, pudo haber determinado en mayor medida la construcción megalítica. Esta variable locacional ha focalizado el esfuerzo de la investigación en torno al fenómeno megalítico, oscilando entre posiciones más especulativas derivadas del importante papel que pudo haber desempeñado el túmulo para la comunidad de los vivos, hasta planteamientos más interpretativos que conciben la visibilidad como la capacidad de las necrópolis para ser perceptibles en el terreno circundante (Carrero Pazos 2017).

Desde estos segundos planteamientos, se analizó la visibilidad del entorno topográfico de las necrópolis a partir de las propuestas de M. Llobera (2006a, 2007). Dicha aproximación toma como punto de partida un cálculo de visibilidad total, que se define como la visibilidad inherente a todas las localizaciones en un paisaje, resultando zonas que son visualmente más prominentes en el sentido de una mayor magnitud visual (Llobera 2003; Llobera et al. 2010). Este análisis calcula la visibilidad acumulada de cada una de las celdas del MDE.

En la zona de estudio la descomposición del MDE generó 1.213 .588 puntos, imposibilitando un cálculo de visibilidad total fuera de un entorno de supercomputación. Por ello, se realizó una aproximación muestral estratificada ${ }^{12}$, creando una red de puntos regulares espaciados cada $500 \mathrm{~m}$ (3.030 puntos en total), y se calculó, para cada uno de ellos, la visibilidad acumulada con r.viewshed.cva ${ }^{13}$ en GRASS GIS 7.0.4 (Fig. 3A). El resultado del cálculo se muestra en la figura $3 \mathrm{~B}$, con un alto porcentaje de túmulos localizados en áreas de visibilidad baja y media (Fig. 3C).

Este análisis no nos permite hablar de preferencias locacionales pues desconocemos si el patrón visual del emplazamiento topográfico tumular se repite en el territorio circundante. Para poder hacerlo acudimos a la Simulación de Monte Carlo, un método basado en la construcción de un rango de distribuciones aleatorias que representan las circunstancias de las que se piensa se desviará la distribución real observada.

${ }_{12}$ Una aproximación similar puede encontrarse en Rodríguez Rellán, C. y Fábregas Valcarce, R.: "Monuments on the move. Assessing megalith's interaction with NW Iberian landscapes". Edición previa de la comunicación presentada a la "Early monumentality and social differentiation in Neolithic Europe: megaliths, societies, landscapes conference (Kiel, Alemania 2015)". Prevista publicación en M. Hinz (ed.): Megaliths, societies and landscapes: early monumentality and social differentiation in Neolithic Europe. Universität zu Kiel.

${ }^{13} \mathrm{https}$ //grass.osgeo.org/grass72/manuals/addons/r.viewshed.cva.html (consulta 12-09-2017). 


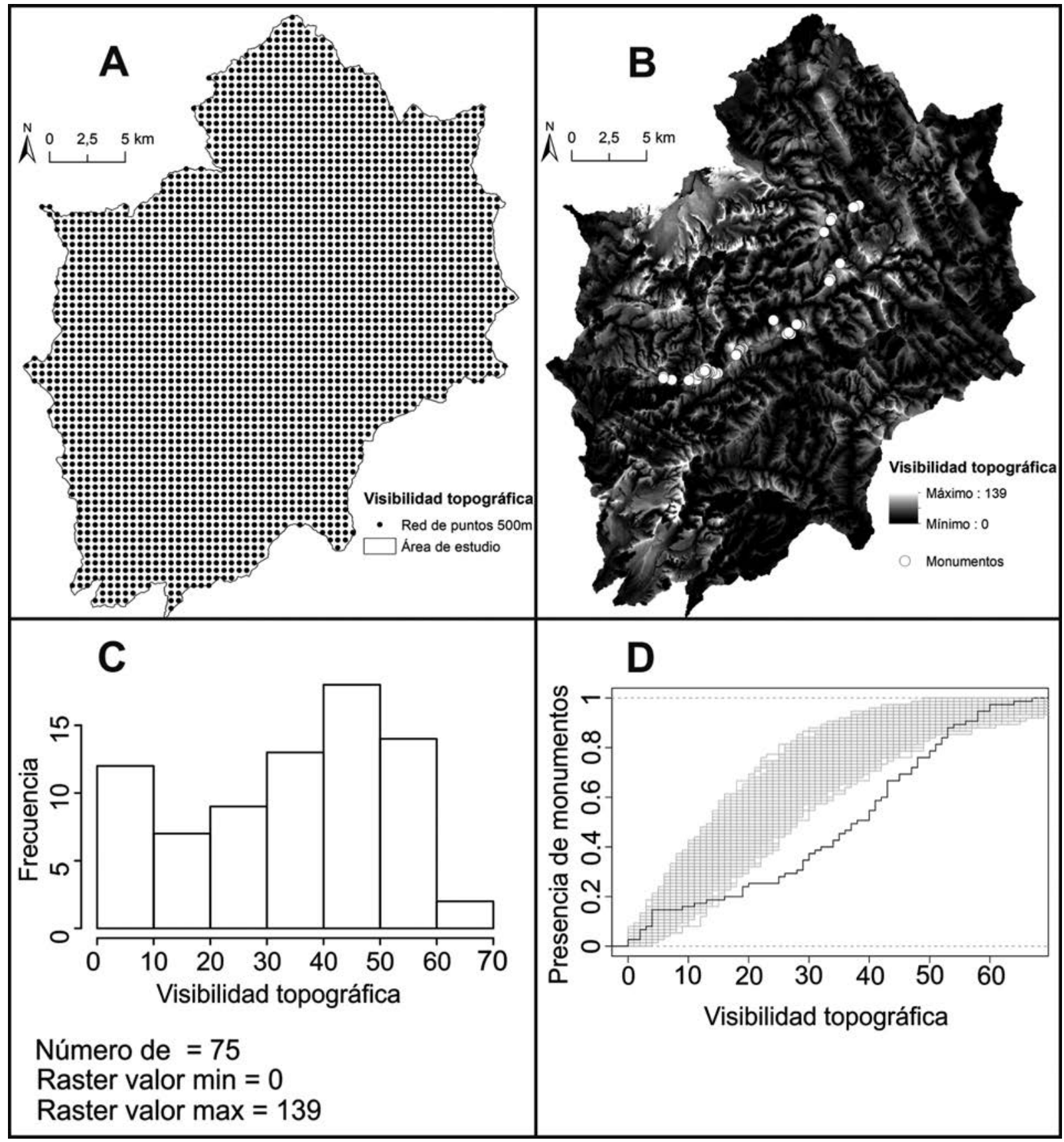

Fig. 3. Aproximación a la visibilidad topográfica del Monte de Santa Mariña y su entorno: A. red regular de puntos distanciados cada $500 \mathrm{~m}$; B. mapa de visibilidad topográfica; C. histograma del cálculo de visibilidad acumulada con los valores para los túmulos megalíticos; D. comparación entre los valores de visibilidad de los monumentos (línea negra) y un conjunto de 999 simulaciones aleatorias distribuidas por la totalidad del área de estudio (rango gris).

La mayor parte de los autores (Manly 1991; Fischer et al. 1997; Drennan y Peterson 2004; Crema et al. 2010; Bevan et al. 2013), utilizan 999 simulaciones aleatorias como el conjunto admisible para aceptar o rechazar la hipótesis nula de aleatoriedad en los datos, obteniendo un valor $p$ significativo cuando $p<=0,05$. En este trabajo, dicha aproximación fue modelizada en $R$ statistics, creando un conjunto de 999 simulaciones 
aleatorias cuya localización no coincide con la de los monumentos (limitados a partir de un buffer de $1 \mathrm{~km}$ ), gracias al uso de un loop. Posteriormente, mediante una distribución de frecuencia acumulada se obtuvo un resultado gráfico del cálculo (Fig. 3D).

La comparación de la distribución real y la resultante de las simulaciones aleatorias, permite observar que el estadístico de los túmulos no coincide con el rango aleatorio en los valores medios. Esto significa que la localización de los mismos en dichas áreas no es aleatoria, actuando la visibilidad como un posible factor locacional que explicaría su distribución específica. Donde la visibilidad topográfica es baja o alta existe igual probabilidad de encontrar túmulos que puntos aleatorios, por lo que dichas localizaciones no parecen ser intencionales.

El estudio de la visibilidad topográfica, tal como ha sido modelizada en este trabajo, nos permite hablar de una localización en áreas específicas del territorio, donde la visibilidad juega un papel importante (hipótesis ya formulada en Criado Boado y Villoch Vázquez 1998), si bien es necesario seguir profundizando.

\subsection{Moundscapes o los túmulos en el paisaje}

Valoramos la relación entre los diferentes conjuntos tumulares y su territorio circundante estudiando, en primer lugar, su impacto visual. Hemos adoptado la aproximación de P. Murrieta Flores (2012), quien adecuó los rangos visuales de T. Higuchi (1983) al caso concreto del megalitismo del sur de la Península Ibérica, definiendo tres umbrales de visibilidad: bajo $(0-478 \mathrm{~m})$, medio $(478-1798 \mathrm{~m})$ y alto $(1.798-$ $3.000 \mathrm{~m})$. A estas estimaciones, se sobrepuso un análisis de visibilidad simple a buffers generados con las distancias anteriores, obteniendo con ello un mapa de visibilidades escalado en función de los umbrales antes definidos. El 16,84\% de la cuenca visual de los túmulos del Monte de Santa Mariña y su entorno se corresponde con rangos visuales bajos, un 55,05\% con rangos medios y un $28,11 \%$ con rangos altos (Fig. 4). Esto sugiere que el monumento funerario tuvo que ser concebido como un hito visible en el territorio. La orientación visual de los monumentos sobre el paisaje se valoró analizando la visibilidad direccional, que permite identificar la orientación específica de la cuenca visual de los monumentos en el paisaje (Wheatley y Gillings 2000). El desarrollo metodológico consiste en crear un ráster de orientaciones, después reclasificado en grados correspondientes a los puntos cardinales y sobre el cual se superpone, como antes, un ráster de visibilidad simple. En nuestro caso (Fig. 4), un 20,99 \% de los monumentos megalíticos descargan su potencial visual hacia el $\mathrm{N}$, un $16,34 \%$ hacia el SE y un $15,56 \%$ hacia el NO, confirmando que la orientación preferencial de las necrópolis es la septentrional (Carrero Pazos y Rodríguez Casal 2015).

Finalmente, y siendo conscientes de los problemas que introducen los análisis de visibilidad simple (Wheatley y Gillings 2002; Conolly y Lake 2006; Paliou 2013), se precisó el cálculo de visibilidades recurriendo al de visibilidades "borrosas" o tipo fuzzy (Fischer 1992, 1993; Ogburn 2006). Este cálculo evalúa el cambio visual de un objeto por su tamaño y distancia del observador definiendo el "nivel de claridad" del objeto. El cálculo fue realizado en $\operatorname{ArcGIS}$ mediante la herramienta fuzzy viewshed creada por A. Rášová (2014: 1), que utiliza la función de pertenencia:

$\mu_{\mathrm{f}}=\frac{1}{1+2\left(\frac{d-b_{1}}{b_{2}}\right)^{2}}$ para $d>b_{1}$; donde $b_{1}$ es la distancia de visibilidad clara, $d$ es la distancia euclidiana y $b_{2}$ es la distancia crítica para que el ojo humano detecte un objeto $b_{2}=\frac{\mathrm{h}}{2 \tan \left(\frac{\beta}{2}\right)}$, donde $h$ es el tamaño del objeto (altura o anchura) y $\beta$ es la agudeza de reconocimiento del ojo humano.

El primer análisis concibió los monumentos como observadores (Fig. 5), identificando las áreas del territorio que son perceptibles desde los mismos. Esto permite observar la existencia de relaciones de visibilidad entre el conjunto de Santa Mariña y las necrópolis septentrionales del Monte Meda, de hecho, las más perceptibles de todo el conjunto. Es interesante destacar que desde los monumentos sólo se percibe el espacio inmediato, con rangos de perceptibilidad bajos en la práctica totalidad de sus cuencas visuales. Esto podría estar indicando que la visibilidad desde el túmulo está más relacionada con el entorno inmediato de las necrópolis que con un juego visual a larga distancia.

En segundo lugar, se calcularon las visibilidades fuzzy para una red de puntos separados cada $500 \mathrm{~m}$ $(\mathrm{n}=3.030)$ (Fig. 6) para identificar las áreas del paisaje más perceptibles y observar en qué medida los túmulos se sitúan en las mismas. La comparación con el rango aleatorio obtenido gracias a la Simulación de Monte Carlo descarta, de nuevo, la localización aleatoria de las mámoas, que parecen ubicadas en zonas de elevada perceptibilidad del territorio (crestas).

En síntesis, los análisis efectuados indicarían que la visibilidad pudo haber desempeñado un papel importante como criterio locacional. Las necrópolis estudiadas mantienen rangos de visibilidad medios, aunque su localización sobre topografías de elevada perceptibilidad en el territorio evidenciaría que la visibilidad debe considerar tanto el monumento como el emplazamiento topográfico. 

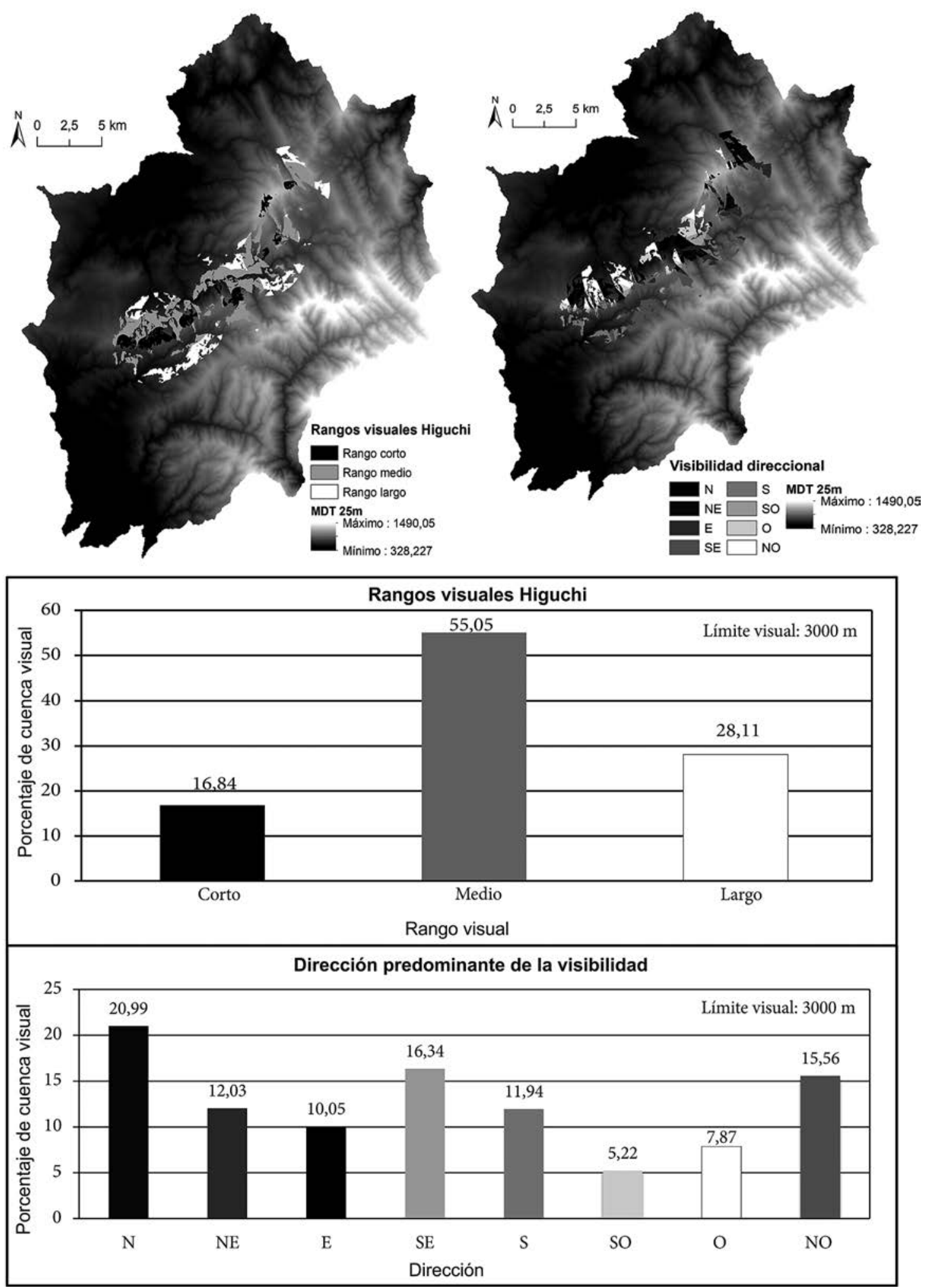

Fig. 4. Aproximación a los rangos visuales Higuchi (siguiendo a Murrieta Flores 2012) y la visibilidad direccional. 

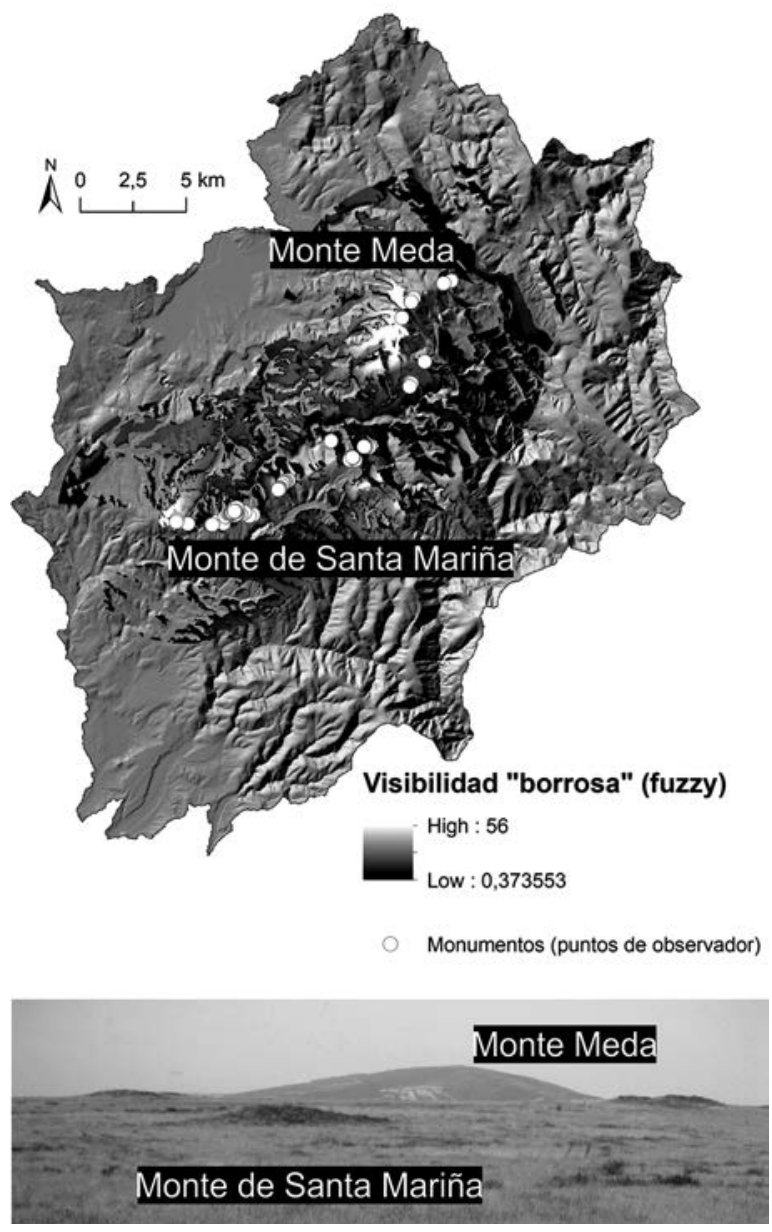

Fig. 5. Visibilidad "borrosa" (fuzzy) calculada con los túmulos megalíticos como puntos de observador, y vista de la necrópolis megalítica del Monte de Santa Mariña hacia el Monte Meda (foto del Grupo de investigación-1520 "Arqueoloxía e ecoloxía do fenómeno tumular e megalítico galego", verano de 2005). Base cartográfica: Centro Nacional de Información Geográfica, con una resolución de $25 \mathrm{~m}$.

\section{UN MODELO DE SIMULACIONES PARA EL ESTUDIO DE LAS RELACIONES ENTRE LA MOVILIDAD Y VISIBILIDAD}

Una vez tenemos analizada tanto la movilidad natural a través del territorio como la visibilidad de los monumentos, es posible elaborar un modelo de simulaciones para valorar las relaciones entre la intensidad de tránsito potencial y la visibilidad de los túmulos megalíticos. Para ello, se llevaron a cabo cuatro aproximaciones (Tab. 2).

Primero se calculó la intensidad visual del territorio próximo a los diferentes conjuntos megalíticos. Para ello se definió un límite isócrono de 30 minutos de marcha
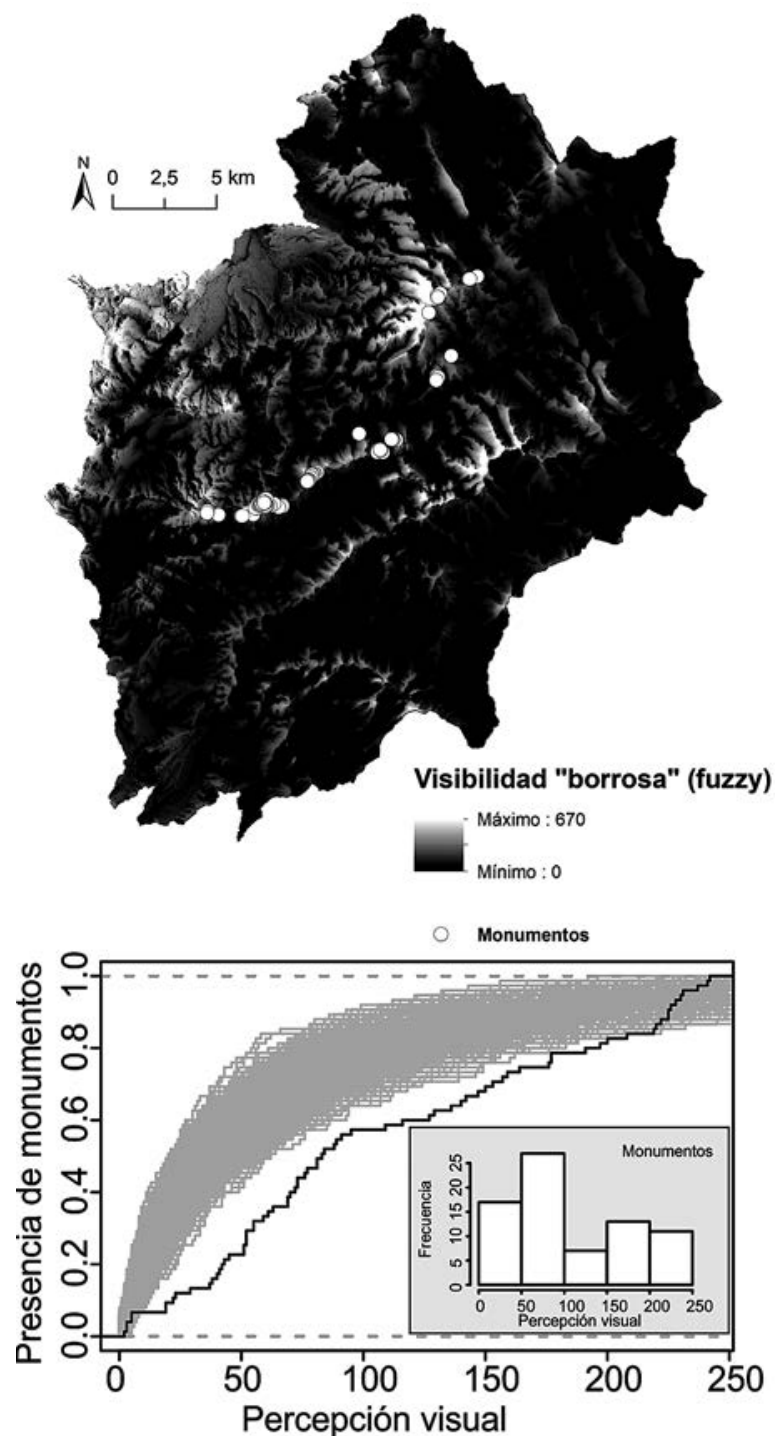

Fig. 6. Arriba: mapa de visibilidad "borrosa" (fuzzy) para un grid de puntos distribuidos cada $500 \mathrm{~m}$ por el territorio del Monte de Santa Mariña y su entorno. Abajo: comparación entre los valores de visibilidad "borrosa" correspondientes a los monumentos (línea negra e histograma) y un conjunto de 999 simulaciones aleatorias distribuidas por la totalidad del área de estudio (rango gris). Base cartográfica: Centro Nacional de Información Geográfica, con una resolución de $25 \mathrm{~m}$.

a pie desde los monumentos, calculado computacionalmente en GRASS GIS 7.0.4 con r.walk. Este límite nos permite valorar la relación visual del territorio circundante con los túmulos o sus áreas de emplazamiento, a partir de la definición de un entorno accesible en un tiempo determinado a los monumentos (Carrero-Pazos 2017) (Tab. 2A). Además se analizó la relación visual entre las rutas de tránsito potenciales y los monumentos 


\begin{tabular}{|c|c|c|c|}
\hline Ref. & Análisis & Método & Resultados \\
\hline $\mathrm{A}$ & $\begin{array}{c}\text { Intensidad visual } \\
\text { topográfica }\end{array}$ & $\begin{array}{c}\text { Visibilidad acumulada } \\
\text { (red de puntos regulares } \\
\text { cada 500 m) }\end{array}$ & $\begin{array}{c}\text { Identificación de las áreas más visibles } \\
\text { del territorio }\end{array}$ \\
\hline $\mathrm{B}$ & $\begin{array}{c}\text { Intensidad visual desde } \\
\text { la red de tránsito potencial }\end{array}$ & $\begin{array}{c}\text { Visibilidad acumulada } \\
\text { (rutas divididas en puntos } \\
\text { cada 500 m) }\end{array}$ & $\begin{array}{c}\text { Identificación del territorio que es visible } \\
\text { desde las rutas de tránsito potenciales }\end{array}$ \\
\hline $\mathrm{C}$ & $\begin{array}{c}\text { Intensidad visual desde } \\
\text { los monumentos }\end{array}$ & $\begin{array}{c}\text { Visibilidad acumulada } \\
\text { desde los monumentos }\end{array}$ & $\begin{array}{c}\text { Identificación del territorio que es visible } \\
\text { desde los monumentos }\end{array}$ \\
\hline $\mathrm{D}$ & $\begin{array}{c}\text { Percepción visual desde } \\
\text { la red de tránsito potencial }\end{array}$ & $\begin{array}{c}\text { Visibilidad fuzzy } \\
\text { (rutas divididas en puntos } \\
\text { cada } 500 \mathrm{~m} \text { ) }\end{array}$ & $\begin{array}{c}\text { Identificación del nivel de perceptibilidad del } \\
\text { territorio desde las rutas de tránsito potenciales }\end{array}$ \\
\hline
\end{tabular}

Tab. 2. Aproximaciones analíticas definidas para el estudio de la relación entre la visibilidad y la movilidad en los conjuntos monumentales seleccionados del Monte de Santa Mariña y su entorno.

(Tab. 2B). La metodología para este cálculo de visibilidad acumulada fue similar a la empleada para la visibilidad topográfica, utilizándose un script de Python creado para GRASS GIS versión 7.0.4 que permite calcular la visibilidad acumulativa con gran cantidad de puntos a la vez. La figura 7 muestra el número de rutas potenciales y su descomposición en puntos cada $500 \mathrm{~m}$, a partir de los que se calculó la visibilidad acumulativa con una altura del observador de 1,65 m y un límite visual de $3.000 \mathrm{~m}$.

Los resultados sugieren una relación visual importante entre las vías de tránsito potencial y los monumentos, algunos de ellos observables desde un elevado número de rutas. Este análisis, sin embargo, no permite identificar los puntos concretos de las rutas de tránsito desde los que pueden observarse los túmulos. Para lograrlo se calculó la visibilidad acumulada desde los propios monumentos (Tab. 2C), identificando las porciones del terreno visible desde los monumentos, y por lo tanto, de las rutas de tránsito ${ }^{14}$ (Fig. 8A). En base a esto, un cálculo de visibilidad binaria simple permite observar las partes específicas de las rutas desde las que es posible divisar los monumentos (Fig. 8B). La figura 9 muestra la cuantificación de este análisis. Se percibe que, en un radio de desplazamiento de media hora, la mayor parte del tránsito mantiene una importante relación visual con los monumentos. Además, siendo más exhaustivos, los monumentos poseen una mayor visibilidad desde las rutas concentradas en los puntos de entrada y salida de las sierras $(59,87 \%)$, así como en la práctica totalidad de los cruces viarios. Ello sugiere que los túmulos podrían haber sido utilizados

${ }^{14}$ El cálculo ha sido realizado en GRASS GIS versión 7.0.4, mediante el addon r.viewshed.cva con una target elevation de 1,65, observer elevation de 0 y un límite visual de $8.000 \mathrm{~m}$. como un marcador territorial, tal como investigaciones anteriores señalaban (véase Martinón-Torres 2001).

Por último, se calcularon las visibilidades fuzzy desde las rutas de tránsito (Tab. 2D), simulando de forma teórica la percepción de una persona que caminara por ellas y observase los monumentos. Para ello se descompuso el vector de rutas en puntos situados cada $500 \mathrm{~m}$, generando un total de 3.450 posiciones de observador. Según los resultados, los monumentos se ubican en zonas de percepción media desde las rutas de tránsito potencial, manteniendo un patrón no aleatorio que, de nuevo, muestra una relación entre las rutas de desplazamiento potencial y la percepción de los monumentos (Fig. 10).

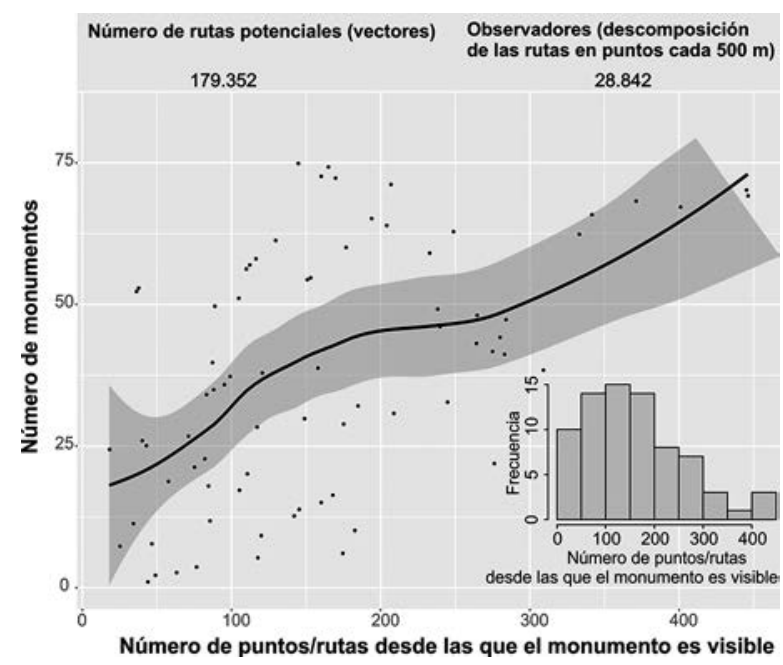

Fig. 7. Comparación entre el número de túmulos megalíticos y el número de puntos/rutas desde los que son visibles en el territorio del Monte de Santa Mariña y su entorno. 

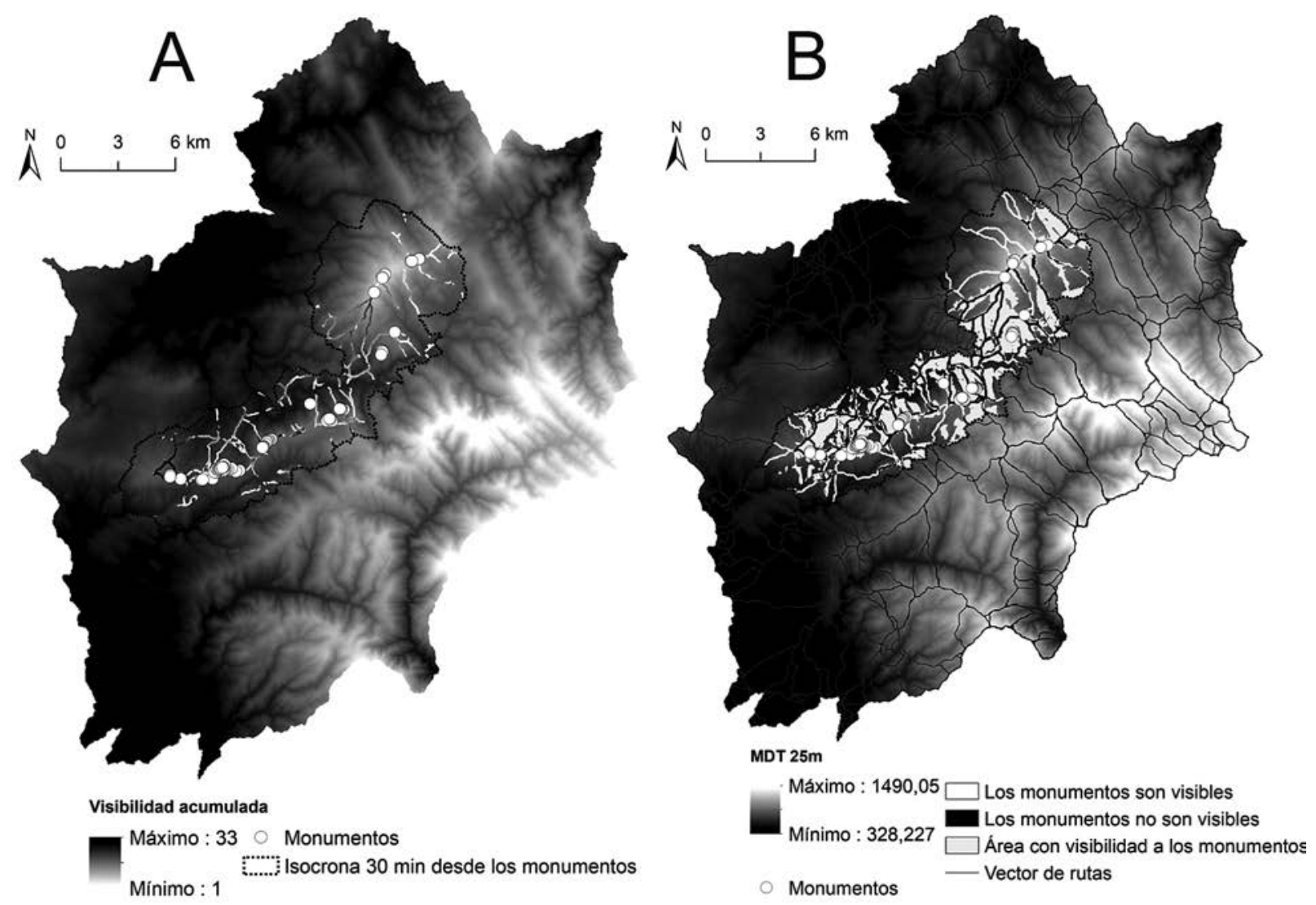

Fig. 8. A. visibilidad acumulada desde las rutas de tránsito potencial hacia el emplazamiento de los monumentos del Monte de Santa Mariña y su entorno, sobre mapa isócrono con media hora de desplazamiento; B. visibilidad binaria para las rutas de tránsito potencial sobre mapa isócrono con áreas desde las que se pueden observar los túmulos megalíticos a media hora de desplazamiento. Base cartográfica: Centro Nacional de Información Geográfica, con una resolución de $25 \mathrm{~m}$.

\section{6. ¿LA VISIBILIDAD COMO FACTOR LOCACIONAL? UNA APROXIMACIÓN A TRAVÉS DE LA SIMULACIÓN DE MONTE CARLO}

Los análisis anteriores permiten valorar la relación entre la movilidad por el territorio y el impacto visual del megalito, pero no desvelan si la visibilidad, como la movilidad, supuso un criterio locacional de primer orden. Esta cuestión puede abordarse examinando la conexión entre el tamaño de la cuenca visual y la localización de los túmulos (Lake y Woodman 2000) ${ }^{15}$.

${ }^{15}$ Somos conscientes de que esta aproximación al análisis de la visibilidad reduce un criterio locacional complejo y heterogéneo a una sola variable - el tamaño de la cuenca visual del megalito-, cuando otros aspectos como los horizontes visuales y los juegos cromáticos influyen en dicho factor. Serán tenidos en cuenta en futuras contribuciones.
El planteamiento metodológico que se propone, basado en la Simulación de Monte Carlo, busca comparar el tamaño de la cuenca visual de los monumentos megalíticos con dos aproximaciones aleatorias que consideran el territorio del área de estudio. La muestra de puntos aleatorios se distribuye en la primera por la totalidad del área de trabajo. En cambio, en la segun$\mathrm{da}$, se fuerza la localización de los puntos en las áreas donde, según el modelo predictivo realizado en nuestro trabajo doctoral (Carrero Pazos 2017: 218 y ss.), poseen valores elevados $\mathrm{y}$, por lo tanto, pueden responder a un patrón de localización megalítico. A partir del análisis locacional se desarrolló un análisis de regresión logística multivariante para comparar la relación entre 11 covariables y la variable dependiente (monumentos megalíticos). Las variables resultantes que predicen la distribución de los monumentos son la geología y los costes de desplazamiento desde las zonas de acumulación de agua potencial y desde las rutas de tránsito. El 


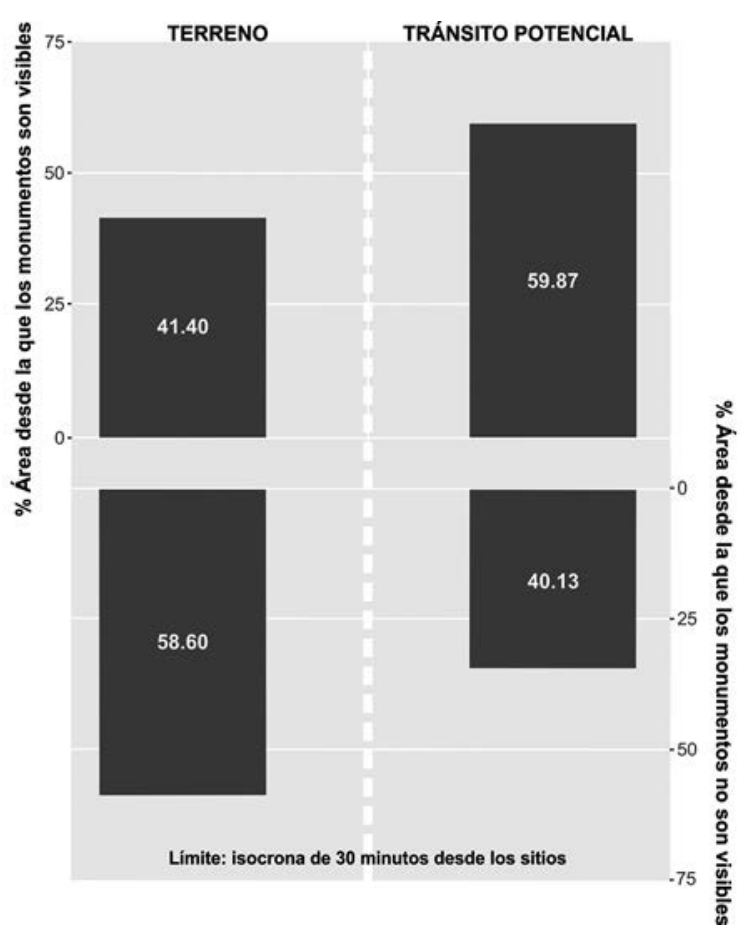

Fig. 9. Porcentaje de área desde la que los túmulos megalíticos del Monte de Santa Mariña y su entorno son visibles o no, para el terreno y las rutas de tránsito potencial.

modelo predictivo fue validado mediante el control de casos (más del $70 \%$ de la muestra de control se sitúa en valores predictivos superiores al 0,5), y el estadístico $G$ de Kvamme (0,701) (Kvamme 1988) (Fig. 11, Tab. 3) ${ }^{16}$.

La comparación estadística partió del trabajo de $\mathrm{M}$. Lake y P. Woodman (2000), empleando, como ya se ha indicado, la Simulación de Monte Carlo. De esta forma podemos determinar, primero, si el tamaño de la cuenca visual de los túmulos es aleatorio o no $\mathrm{y}$, en segundo lugar, si el tamaño de la cuenca visual de los túmulos en el área de estudio pudo haber sido un factor importante en su ubicación. Esta correlación, tradicionalmente defendida para el conjunto del fenómeno megalítico en Galicia (véase Criado Boado 1984 como síntesis), no significa causalidad.

Fueron definidas dos muestras aleatorias para comparar el tamaño de las respectivas cuencas visuales con la de los túmulos megalíticos $(\mathrm{n}=75)$ (material complementario AC2): la primera creada bajo condiciones aleatorias completas (Poisson) $(\mathrm{n}=74.925)$ (material

\footnotetext{
16 Para los pormenores del modelo predictivo realizado para esta área y resumidos en la figura 13, véase Carrero Pazos 2017: 218 y ss. El uso de estos resultados se justifica en la definición de aquellas áreas de la zona de estudio que comparten los valores predictivos que se derivan de la localización de los monumentos.
}

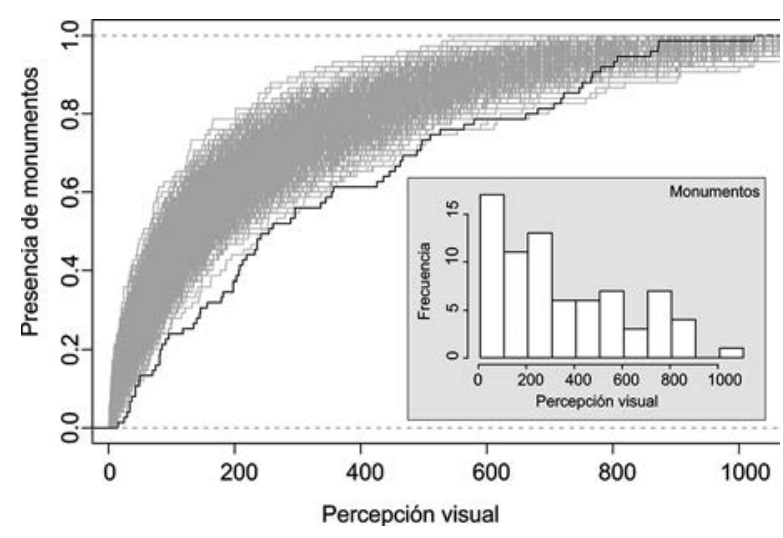

Fig. 10. Simulación de Monte Carlo sobre visibilidad fuzzy, calculada desde las rutas de tránsito potencial. La línea negra y el histograma indican los valores de los monumentos, mientras que el rango gris muestra el resultado de 999 simulaciones aleatorias distribuidas por la totalidad del área de estudio.

\begin{tabular}{|c|c|c|}
\hline $\begin{array}{c}\text { Variables } \\
\text { de primer } \\
\text { orden }\end{array}$ & Descripción & $\begin{array}{c}\text { Modelo } \\
\text { de regresión } \\
\text { generalizado }\end{array}$ \\
\hline \multirow{4}{*}{$\begin{array}{c}\text { Factores } \\
\text { físicos y } \\
\text { orográficos }\end{array}$} & Altitud & \multirow{2}{*}{ Predicción baja } \\
\hline & Pendiente & \\
\hline & Geología & \multirow{3}{*}{ Predicción alta } \\
\hline & $\begin{array}{c}\text { Distancia } \\
\text { desde zonas de } \\
\text { acumulación } \\
\text { de agua }\end{array}$ & \\
\hline \multirow{3}{*}{$\begin{array}{c}\text { Factores } \\
\text { potenciales }\end{array}$} & $\begin{array}{l}\text { Distancia desde } \\
\text { rutas de tránsito } \\
\text { potencial }\end{array}$ & \\
\hline & $\begin{array}{l}\text { Prominencia } \\
\text { topográfica }\end{array}$ & \multirow{2}{*}{ Predicción baja } \\
\hline & $\begin{array}{l}\text { Visibilidad } \\
\text { topográfica }\end{array}$ & \\
\hline
\end{tabular}

Tab. 3. Modelo predictivo para el análisis locacional de los conjuntos monumentales seleccionados en el Monte de Santa Mariña y su entorno. Resultados del modelo de regresión generalizado (Carrero Pazos 2017: 509).

complementario AC3), y la segunda constreñida espacialmente a las áreas con alto valor predictivo, extraídas del modelo realizado $(n=74.925)$ (material complementario $\mathrm{AC} 4$ ).

Los análisis fueron realizados en GRASS GIS en su versión 7.0.3, con el módulo r.cva ${ }^{17}$, utilizando un

17 http://www.ucl.ac.uk/ tcrnmar/GIS/r.cva_v5x_man.html (consulta $17-10-2017)$. 


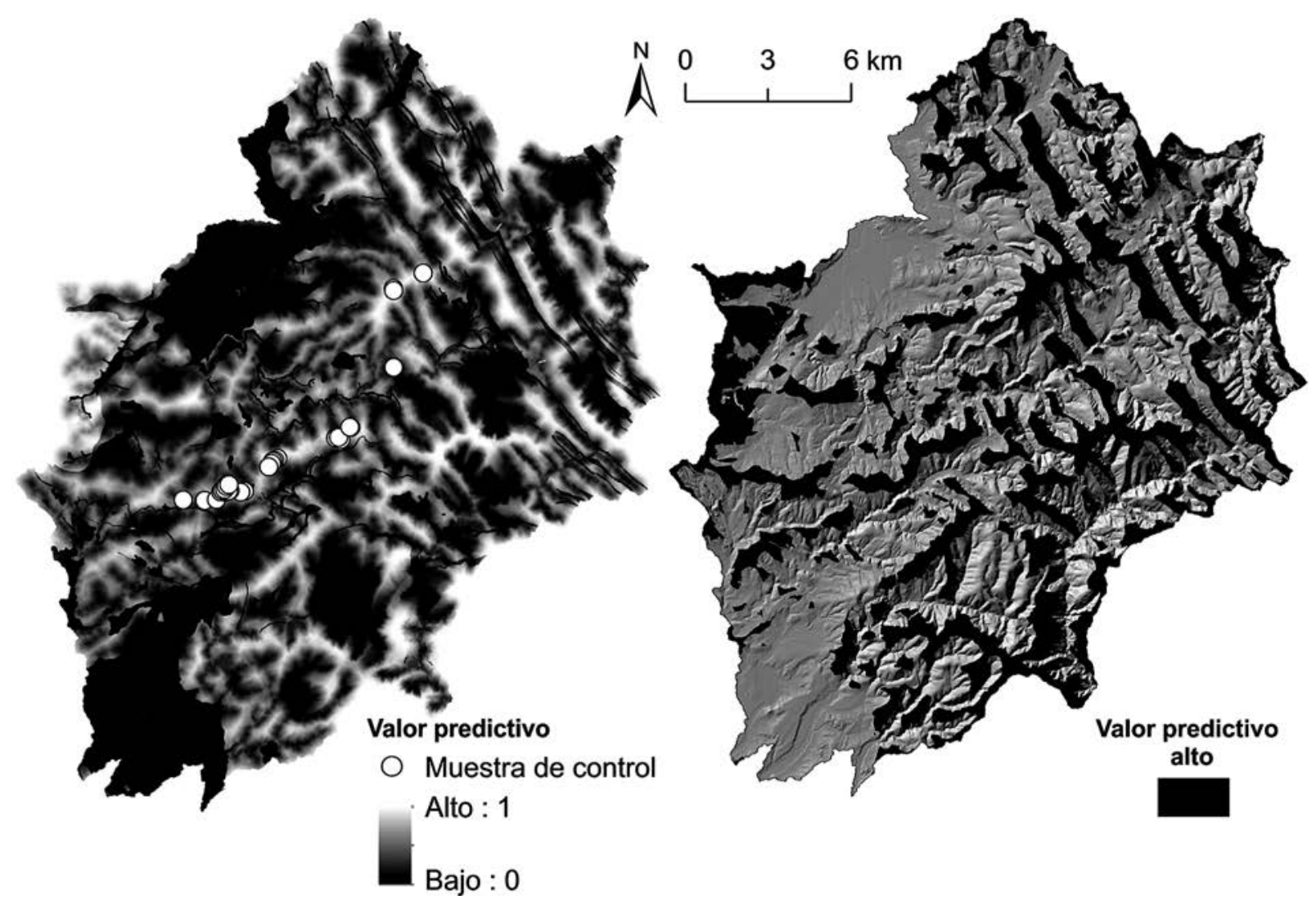

Fig. 11. Resultados del modelo predictivo que permite identificar las áreas del territorio con un patrón similar al de los túmulos megalíticos (Carrero Pazos 2017: 463). Base cartográfica: Centro Nacional de Información Geográfica, con una resolución de $25 \mathrm{~m}$.

MDE de $25 \mathrm{~m}$ de resolución. Como en los cálculos anteriores, la resolución elegida está justificada por el elevado esfuerzo computacional que acarrea el uso de resoluciones más precisas.

Partiendo del ráster de visibilidad topográfica creado anteriormente, se calculó el tamaño de la cuenca visual de cada punto con una altura del observador de $1,65 \mathrm{~m}$ y un límite visual de $4.000 \mathrm{~m}$. Posteriormente, empleando r.stats, se extrajo el tamaño de la cuenca visual $\left(\mathrm{km}^{2}\right)$ para cada uno de los puntos tanto de la muestra arqueológica como de las aleatorias. Finalmente, dos pruebas estadísticas en $R$ Statistics se orientaron a conocer la relación entre el tamaño de la cuenca visual de la distribución arqueológica con respecto al tamaño de la de las dos muestras aleatorias.

Para visualizar dicha comparación utilizamos el gráfico de frecuencia acumulada (Fig. 12). Sus resultados muestran que el tamaño de la cuenca visual de los túmulos no parece alejarse del conjunto de ambas muestras aleatorias. La muestra no constreñida por los factores de primer orden (modelo predictivo) mantendría una mayor diferencia significativa en los rangos medios, al situarse cerca de los límites del intervalo aleatorio (Fig. 12A).

Los gráficos de frecuencia acumulada permiten analizar el grado de significación de forma visual, pero no proporcionan una medida de significación (valor $p$ ). Para obtenerlo, debemos calcular, en primer lugar, la diferencia absoluta entre la frecuencia acumulada del tamaño de la cuenca visual de los monumentos y la del tamaño de las cuencas visuales de todas las muestras aleatorias, utilizando la prueba de Kolmogorov Smirnov (KS). Los resultados de la muestra aleatoria sin condicionar espacialmente, resumidos en los diagramas de caja (Fig. 13A), indican valores $p$ significativos en su mayor parte (615 casos con valores menores a $0,05=61,56 \%$ de la muestra aleatoria). Este dato permite afirmar que los tamaños de las cuencas visuales de los túmulos y de los puntos aleatorios difieren entre sí. Por otra parte, los resultados de la muestra aleatoria constreñida por el modelo predictivo (Fig. 13B) indican valores no significativos ( 25 casos por debajo de $0,05=2,50 \%$ de la muestra aleatoria). Por ello no se puede afirmar que el tamaño de la cuenca visual 

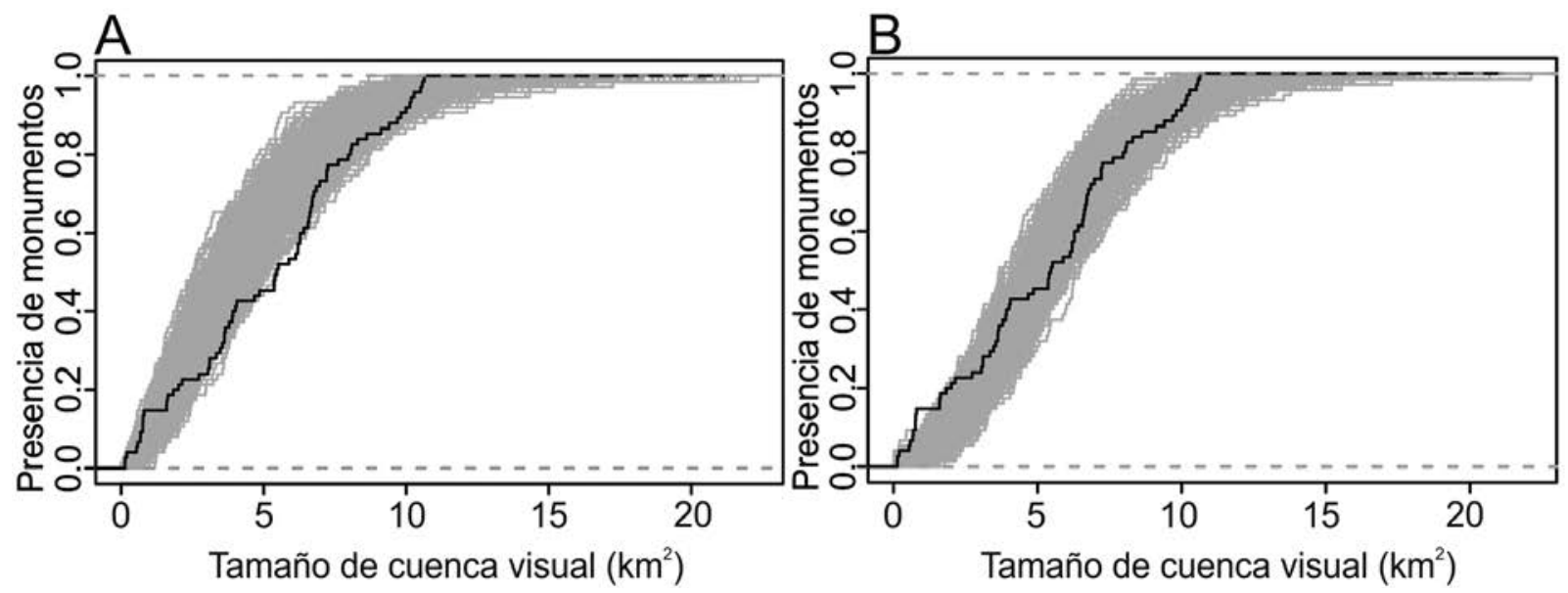

Fig. 12. Distribución de frecuencias acumuladas para el tamaño de la cuenca visual. Túmulos megalíticos (línea negra). 999 Simulaciones aleatorias (rango gris). Muestras aleatorias: A. sin condicionar; B. constreñida.

de los túmulos megalíticos difiera del tamaño de los puntos aleatorios condicionados por el posible patrón de localización megalítico.

La clasificación de medias (Lake y Woodmann 2000: 501) es un segundo procedimiento estadístico escogido para estimar la probabilidad de que la media del tamaño de la cuenca visual de la muestra arqueológica proceda de la misma población que las distribuciones simuladas. De esta forma, si dividimos la posición de la muestra real en una clasificación de las medias por la posición de la muestra arqueológica -1.000 , ya que las 999 anteriores se corresponden con las simu-
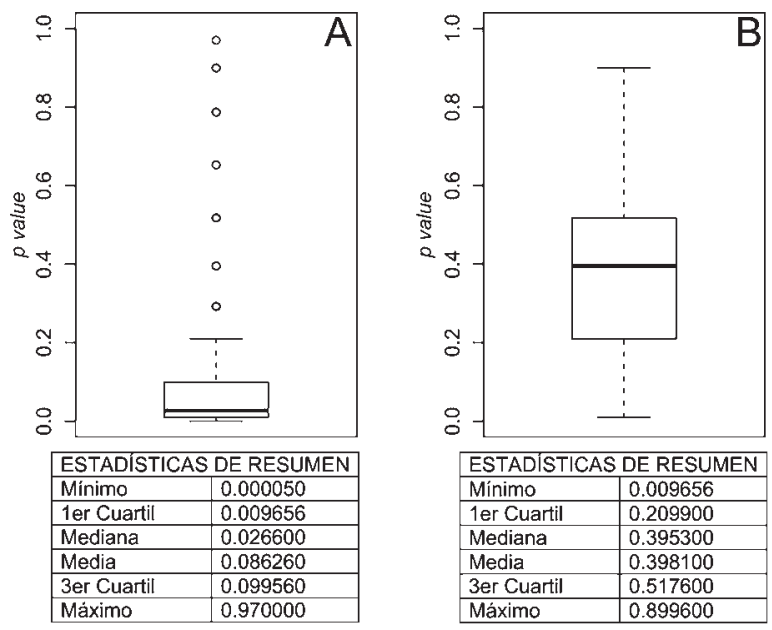

Fig. 13. Visibilidad en los patrones de localización de los túmulos megalíticos del Monte de Santa Mariña y su entorno. Diagramas de caja y bigotes con los valores de significación del test de Kolmogorov Smirnov para las muestras aleatorias: A. sin condicionar; B. constreñida. laciones aleatorias-, obtendremos la probabilidad de rechazar de forma incorrecta la hipótesis nula, según la cual, las medias del tamaño de la cuenca visual de ambas muestras proceden de la misma población de cuencas visuales.

Por lo tanto, mediante estadística descriptiva (clasificación de las medias), observamos que en el caso de la población aleatoria sin constreñir, la muestra arqueológica se sitúa en la decimoquinta posición de un total de 1.000 simulaciones, resultando $15 / 1.000=0,015$. Este dato permite plantear que el tamaño de la cuenca visual de los túmulos es significativamente diferente $(p$ value $<0,05)$ al de los puntos aleatorios localizados en el área de estudio. En las distribuciones aleatorias determinadas por el modelo predictivo, la media del tamaño de las cuencas visuales se sitúa en el número de 727 de un total de 999 simulaciones, lo que supone $727 / 1 \cdot 000=0,727$. Este valor nos indica que la media del tamaño de la cuenca visual de la muestra arqueológica no difiere significativamente del de la muestra aleatoria.

La interpretación de las dos aproximaciones estadísticas realizadas junto con la Simulación de Monte Carlo debe llevarnos a valorar de forma más objetiva la importancia de la visibilidad en los patrones de localización de los túmulos megalíticos gallegos. Los resultados de la comparación de la muestra arqueológica con la aleatoria sin condicionar indican diferencias importantes en lo que al tamaño de la cuenca visual se refiere. Esto no debiera sorprender dadas las notables diferencias entre ambas muestras (Fig. 14) y permite concluir que el tamaño de la cuenca visual de los túmulos no es aleatorio, es decir, que hay diferencias con respecto al entorno topográfico. No obstante, dichas diferencias no pueden entenderse en términos de selección territorial, pues se deben a la comparación de territorios heterogéneos. 

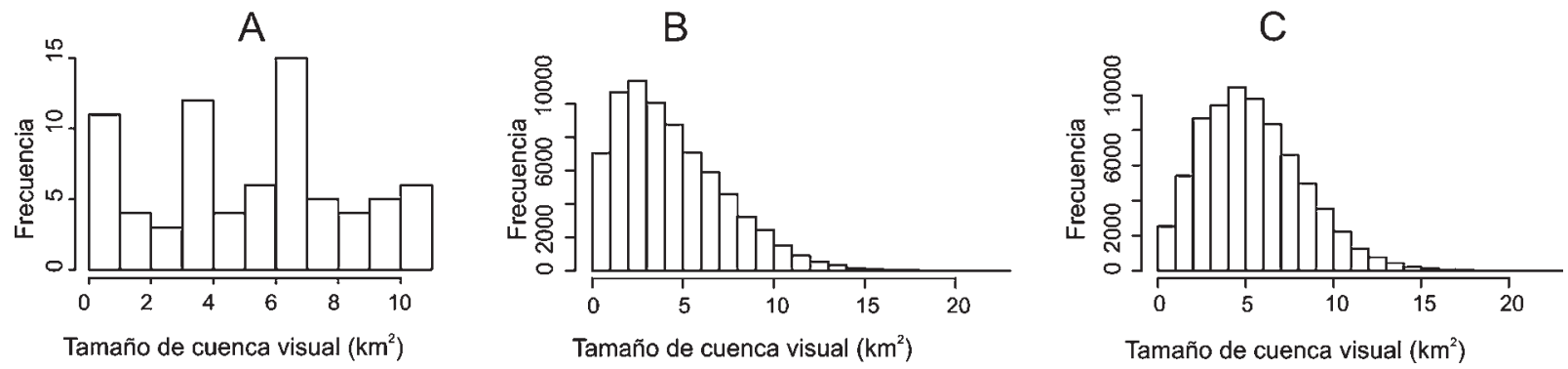

Fig. 14. Histogramas del tamaño de la cuenca visual del Monte de Santa Mariña y su entorno: A. túmulos megalíticos; B. población aleatoria sin condicionar; C. población aleatoria constreñida.

Ambas poblaciones muestran una menor variabilidad si se compara el tamaño de la cuenca visual de los monumentos con el de los territorios que responden a criterios de localización megalíticos (Fig. 14A y 14C). Ello también se infiere de los resultados del test de KS y de la media de los valores de las cuencas visuales de las muestras y parece evidenciar una correlación entre el tamaño de la cuenca visual y los factores de localización que definen el modelo predictivo.

No obstante, estos resultados tampoco permiten confirmar una selección de enclaves determinada por el tamaño de la cuenca visual, pues los territorios cercanos a las necrópolis megalíticas que comparten los mismos factores de localización, poseen cuencas visuales de tamaño similar. El emplazamiento de las necrópolis megalíticas del Monte de Santa Mariña mantiene, por lo tanto, las mismas regularidades en lo que al tamaño de la cuenca visual se refiere que sus territorios adyacentes. Ello excluye una selección del espacio funerario por parte de las comunidades neolíticas en función del tamaño de la cuenca visual de sus monumentos.

\section{CONCLUSIONES Y PERSPECTIVAS EN LA INVESTIGACIÓN}

La visibilidad ha sido el factor locacional que mayor atención ha recibido en Galicia por parte de la investigación sobre el fenómeno megalítico, a partir de una serie de trabajos sobre las relaciones visuales entre el megalito y su medio circundante en la Península del Barbanza (Criado Boado y Villoch Vázquez 1998).

Esta variable ha sido considerada como criterio decisivo en la ocupación megalítica de Galicia y otras áreas del Occidente europeo (véase García et al. 2006; López-Romero 2007; Bourgeois 2013; Murrieta Flores 2014; Rášová 2014; Cerrillo Cuenca y Liceras Garrido 2016). Ahora bien, en Galicia dicho factor se ha centrado en exclusiva en el túmulo, haciendo hincapié en las regularidades visuales existentes dentro de una necrópolis (cfr. Criado Boado 2012) sin tomar en consideración el territorio que alberga los monumentos. Además las interpretaciones suelen articularse a partir del "sentido común" (Lake y Woodman 2003: 690), "descripciones con un alto nivel de detalle y profundidad que más que resolver sirven para formular preguntas con gran significancia y atino. En el peor de los casos, se utilizan para generar interpretaciones nacidas más de la especulación que de cualquier argumento que se haya podido construir" (Llobera 2006b: 110-111). Esta tendencia parece haber provocado un sobredimensionamiento de la relevancia del impacto visual del túmulo como configurador del territorio neolítico. Ello, en vista de los resultados obtenidos en este y otros trabajos (Rodríguez Rellán y Fábregas Valcarce 2015), quizás deba ser matizado en función de las áreas de estudio como sucede con otros criterios de localización, caso de la movilidad ( $c f r$. Carrero Pazos 2017). ${ }^{18}$

Como han demostrado los análisis de los monumentos megalíticos del Monte de Santa Mariña y su entorno, en esta zona específica de Galicia, no suelen emplazarse en las áreas más perceptibles del territorio, sino en otras de intensidad visual media. La mayor parte de los conjuntos tumulares de la zona analizada descargan su potencial visual en dirección norte, existiendo evidentes interrelaciones visuales entre los distintos monumentos. No obstante, la importancia de la visibilidad parece aumentar analizada en conexión con el desplazamiento por el territorio, con umbrales de perceptibilidad elevados en los itinerarios de ascenso y descenso desde/hacia los emplazamientos funerarios, siendo bajos en las zonas más deprimidas.

La visibilidad de los monumentos parece relacionarse, por lo tanto, con las vías de desplazamiento natural, aunque no podemos extrapolar dicha tendencia al conjunto del territorio ni del fenómeno, ya que ni los monumentos ni los emplazamientos megalíticos se aprecian en un porcentaje elevado de lugares.

\footnotetext{
${ }_{18}$ Véase n. 7.
} 
Por otra parte, el uso de la Simulación estadística de Monte Carlo ha permitido profundizar en el estudio de la visibilidad como criterio locacional en el Monte de Santa Mariña y su entorno, teniendo en cuenta las regularidades obtenidas de la comparación de la cuenca visual de los monumentos con las de las dos poblaciones aleatorias ubicadas en zonas aledañas. Dicha aproximación debe ser entendida como un intento más que deberá ser desarrollado y matizado en posteriores trabajos por la gran complejidad analítica de esta variable. Los resultados obtenidos en Santa Mariña y su entorno deben entenderse en un contexto discursivo más amplio, ya que las dinámicas de movilidad y visibilidad aquí descritas trascienden el caso y suponen una aportación a la interpretación de un fenómeno común a otras regiones de Galicia, la Península Ibérica y la fachada atlántica europea. La evidencia empírica presentada nos permite concluir que el factor visual de los monumentos megalíticos del Monte de Santa Mariña y su entorno no es aleatorio. Mantiene un patrón específico y significativo, si bien la posible elección de los emplazamientos tumulares en esta área de estudio no parece haber tenido relación con una selección territorial específica basada en el tamaño de las cuencas visuales. Esto no debe extrañar, pues estamos ante paisajes con diferentes niveles de percepción monumental caracterizados por una importante variedad de situaciones basadas en elementos topogeográficos (Carrero Pazos s. f.), donde el monumento y la necrópolis habrían jugado un papel fundamental, y donde la visibilidad del túmulo y del megalito parece haber estado vinculada a una movilidad elevada de los grupos humanos neolíticos.

Estas conclusiones refuerzan las hipótesis defendidas para otras zonas europeas y peninsulares donde el megalitismo está documentado (p. ej. Whitley y Hicks 2003; Murrieta Flores 2012; ${ }^{19}$ ). Ello sugiere que podríamos estar ante una característica común al conjunto de este fenómeno y que el papel del túmulo como configurador del paisaje neolítico debe ser entendido tanto de forma estática (el megalito como elemento demarcador) como en relación con el desplazamiento general por el territorio. En ese desplazamiento el túmulo incrementa su protagonismo al ser potenciado mediante la visibilidad, haciendo partícipe al difunto de la vida de los vivos, y reforzando con ello el vínculo comunitario a través del recuerdo y la memoria.

\section{AGRADECIMIENTOS}

El Prof. Antón A. Rodríguez Casal nos cedió los datos arqueológicos del fenómeno tumular del Monte

19 Véase n. 12 de Santa Mariña y su entorno, junto con las fotografías de los monumentos, reproducidas aquí. Dichas fotos proceden de la base de datos del grupo de investigación "Arqueoloxía e ecoloxía do fenómeno tumular e megalítico galego" (GI-1520, Universidad de Santiago de Compostela) que desarrolló el proyecto de investigación "Megalitismo e xeoloxía. Arqueoloxía e ecoloxía da cultura dolménica na provincia de Lugo" (1994-1996), subvencionado por la Xunta de Galicia (IP: Antón A. Rodríguez Casal). Además agradecemos a David Espinosa Espinosa la revisión de la redacción de este manuscrito, que contribuyó a una mayor claridad expositiva, y a Carlos Rodríguez Rellán los diferentes consejos en relación al análisis de movilidad.

Tanto el código de $R$ Statistics como el script de Python utilizado para el cálculo de las visibilidades acumuladas fueron redactados durante varios cursos del MSc in Computational Archaeology: GIS, Data Science and Complexity (Institute of Archaeology, $U C L$ ), bajo la supervisión de los Prof. Andrew Bevan y Mark Lake, a quienes corresponde su autoría y a quienes agradecemos su ayuda.

Finalmente, los comentarios y observaciones de los revisores anónimos han enriquecido y mejorado la versión definitiva de este trabajo.

\section{BIBLIOGRAFÍA}

Bello Diéguez, J. M.; Criado Boado, F. y Vázquez Varela, J. M. 1982a: "Sobre la cultura megalítica y los caminos antiguos en Galicia". El Museo de Pontevedra 26: 143-164.

Bello Diéguez, J. M.; Criado Boado, F. y Vázquez Varela, J. M. 1982b: "Megalitismo y medio físico en el Noroeste de la Península Ibérica: estado de la cuestión y perspectivas". Zephyrus XXXIV-XXXV: 109-118.

Bello Diéguez, J. M.; Criado Boado, F. y Vázquez Varela, J. M. 1987: La cultura megalítica de la provincia de la Coruña y sus relaciones con el marco natural: implicaciones socio-económicas. Excma. Diputación provincial de A Coruña. A Coruña.

Bevan, A. 2011: "Computational models for understanding movement and territory". En V. Mayoral Herrera y S. Celestino Pérez (eds.): Tecnologías de información geográfica y análisis arqueológico del territorio. Actas del V Simposio Internacional de Arqueología de Mérida. Anejos de Archivo Español de Arqueología. CSIC. Instituto de Arqueología. Mérida: 384-393.

Bevan, A.; Crema, E.; Xiuzhen, L. y Palmisano, A. 2013: "Intensities, interactions and uncertainties: some new approaches to archaeological distributions". En A. Bevan y M. Lake (eds.): Computational approaches to archaeological landscapes. Left Coast Press. Walnut Creek, California: 27-52

Bevan, A. y Wilson, A. 2013: "Models of settlement hierarchy based on partial evidence". Journal of Archaeological Science 40: 2415-2427. https://doi.org/10.1016/j.jas.2012.12.025

Bradley, R. 1998: The significance of monuments. On the shaping of human experience in neolithic and bronze age Europe. Routledge. London.

Bourgeois, J. 2013: Monuments on the horizon: the formation of the barrow landscape throughout the $3 r d$ and 2 nd millennium $B C$. PhD Dissertation. Leiden University. Leiden. http://hdl.handle.net/1887/20381

Cara Barrionuevo, L. y Rodríguez López, J. M. 1987: “Trashumancia ganadera y megalitos. El caso de valle medio-bajo del río Andarax (Almería)". En Crónica del XVIII Congreso Arqueológico Nacional (Las Palmas de Gran Canaria, Santa Cruz de Tenerife 1987): 235-48. Zaragoza. 
Carrero Pazos, M. 2017: El fenómeno tumular y megalítico en Galicia. Aportaciones desde los Sistemas de Información Geográfica y la Estadística Espacial para el estudio de los patrones de localización. Tesis Doctoral. Universidade de Santiago de Compostela. Santiago de Compostela. https://www.educacion.gob.es/teseo/mostrarRef.do?ref=1470963 (consulta 12-12-2017)

Carrero Pazos, M. s. f.: "Density, intensity and clustering patterns in the spatial distribution of Galician megaliths (NW Iberian Peninsula)". Archaeological and Anthropological Sciences.

https://doi.org/10.1007/s12520-018-0662-2. Forthcoming [Online 6 Jun 2018]

Carrero Pazos, M. y Rodríguez Casal, A. A. 2015: “Definiendo patrones de emplazamiento del Megalitismo gallego: la necrópolis del Monte de Santa Mariña como modelo". Sémata, Ciencias Sociais e Humanidades 27: 299-321.

Carrero Pazos, M. y Vilas Estévez, B. 2016: “The possibilities of the aerial LiDAR for the detection of Galician megalithic mounds (NW of the Iberian Peninsula). The case of Monte de Santa Mariña, Lugo". En S. Campana, R. Scopigno, G. Carpentiero y M. Cirillo (eds.): Keep the Revolution Going. Proceedings of the 43rd Annual Conference on Computer Applications and Quantitative Methods in Archaeology. Archaeopress. Oxford: 901-908.

Carrero Pazos, M.; Vilas Estévez, B.; Romaní Fariña, E. y Rodríguez Casal, A. A. 2014: "La necrópolis del Monte de Santa Mariña revisitada: aportaciones del LiDAR aéreo para la cartografía megalítica de Galicia". Gallaecia 33: 39-57.

Castillo López, Á. del 1927: "Un depósito de hachas del bronce de talón". Boletín de la Real Academia Galega.

Cerrillo Cuenca, E. 2007: "Recorriendo un territorio desaparecido: restitución fotogramétrica y análisis del paisaje de la necrópolis prehistórica del Vado de Alconétar". En V. Mayoral Herrera y S. Celestino Pérez (eds.): Tecnologías de información geográfica y análisis arqueológico del territorio. Actas del V Simposio Internacional de Arqueología de Mérida. CSIC. Mérida: 145-159.

Cerrillo Cuenca, E. y Liceras Garrido, R. 2016. “"Cuencas visuales borrosas' de sepulcros megalíticos. Una aplicación al conjunto de megalitos del entorno de Alconétar". Arpi. Arqueología y Prehistoria del Interior peninsular 05: 04-26.

Chapman, R. 1979: "Transhumance and megalithic tombs in Iberia". Antiquity 53: 150-52. https://doi.org/10.1017/s0003598x00109214

Conolly, J. y Lake, M. 2006: Geographic Information Systems in Archaeology. Cambridge University Press. Cambridge.

Crema, E.; Bevan, A. y Lake, M. 2010: "A probabilistic framework for assessing spatio-temporal point patterns in the archaeological record". Journal of Archaeological Science 37: 1118-1130. https://doi.org/10.1016/j.jas.2009.12.012

Criado Boado, F. 1984: "El tercer factor o la lógica oculta del emplazamiento de los túmulos megalíticos gallegos". Cuadernos de Estudios Gallegos 35: 7-18

Criado Boado, F. 1988: "Arqueología del paisaje y espacio megalítico en Galicia”. Seminario sobre Arqueología Espacial (Tomar 1988). Arqueología Espacial 12: 61-117.

Criado Boado, F. 1989: "Megalitos, espacio, pensamiento". Trabajos de Prehistoria 46: 75-98. https://doi.org/10.3989/tp.1989.v46.i0.588

Criado Boado, F. 1993: "Visibilidad e interpretación del registro arqueológico". Trabajos de Prehistoria 50: 39-56. https://doi.org/10.3989/tp.1993.v50.i0.488

Criado Boado, F. 2012: Arqueológicas. La razón perdida. Bellaterra. Barcelona.

Criado Boado, F.; Fábregas Valcarce, R. y Vaquero Lastres, J. 1990-1991: "Concentraciones de túmulos y vías naturales de acceso al interior de Galicia”. Portugalia XI-XII: 27-38.

Criado Boado, F. y Grajal Blanco, M. 1981: "Relación entre la distribución de mámoas y el medio físico de la zona de Sobrado-Curtis". Brigantium 2: 7-26.

Criado Boado, F. y Vaquero Lastres, J. 1991: “El fenómeno megalítico y tumular: formas diversas de pasado monumental". En F. Criado Boado (ed.): Arqueología del paisaje. El área Bocelo-Furelos entre los tiempos paleolíticos y medievales. Xunta de Galicia. A Coruña: 129-146.

Criado Boado, F. y Vaquero Lastres, J. 1993: "Monumentos, nudos en el pañuelo. Megalitos, nudos en el espacio: Análisis del emplazamiento de los monumentos tumulares gallegos". Espacio, Tiempo y Forma, Serie I, Prehistoria y Arqueología 6: 205-248.

Criado Boado, F. y Villoch Vázquez, V. 1998: "La monumentalización del paisaje: percepción actual y sentido original en el Megalitismo de la Sierra de Barbanza (Galicia)". Trabajos de Prehistoria 55 (1): 63-80. https://doi.org/10.3989/tp.1998.v55.i1.317

Davidson, I. 1980: "Transhumance, Spain and ethnoarchaeology". Antiquity 54: 144-47. https://doi.org/10.1017/s0003598x00103035

Díaz Sanjurjo, M. 1904: "Los caminos antiguos y el itinerario n ${ }^{\circ} 18$ de Antonino en la provincia de Orense". Boletín de la Comisión de Monumentos Históricos y Artísticos de Orense II: 37-43.

De Reu, J. 2012: Land of the dead: a comprehensive study of the Bronze Age burial landscape in north-western Belgium. Ghent University Press. Ghent.

Drennan, R. D. y Peterson, C. E. 2004: “Comparing archaeological settlement systems with rank-size graphs: a measure of shape and statistical confidence". Journal of Archaeological Science 31: 533-549. https://doi.org/10.1016/j.jas.2003.10.002

Eguileta Franco, J. M. a 1997: "Megalitos y coordenadas espaciales en la Baixa Limia: ¿una neolitización tardía en la Galicia interior?”. En A. A. Rodríguez Casal (ed.): O neolítico Atlántico e as orixes do megalitismo. Actas do Coloquio Internacional (Santiago de Compostela 1996): 553-561. Santiago de Compostela.

Eguileta Franco, J. M. ${ }^{a}$ 1999: A Baixa Limia na Prehistoria Recente. Arqueoloxía dunha paisaxe na Galicia interior. Excma. Diputación Provincial de Ourense. Ourense.

Eguileta Franco, J. M. ${ }^{\text {a }}$ 2000: "Megalitos e tránsito: Aproximación ó estudio de vías de comunicación prehistóricas". Lethes. Cadernos Culturais do Limia 2: 46-61.

Ehlschlaeger, C. 1989: "Using the $\mathrm{A}^{\mathrm{T}}$ Search Algorithm to develop hydrologic models from digital elevation data". En Proceedings of International Geographic Information Systems (IGIS) Symposium '89: global systems science - an effective response to human needs. March 18 \& 19, 1989, Baltimore, Maryland. E. H. Pechan and Associates. Springfield, VA: 275-281. https://www.researchgate.net/publication/243781937_Using_the_AT_search_algorithm to_develop_hydrologic_models_from_digital_elevation_data (consulta 22-11-2017)

Fábrega-Álvarez, P.; Fonte, J. y González García, F.J. 2011: “Las sendas de la memoria. Sentido, espacio y reutilización de las estatuas-menhir en el noroeste de la Península Ibérica". Trabajos de Prehistoria 68, 2: 313-340. https://doi.org/10.3989/tp.2011.11072

Fábregas Valcarce, R. 2010 [1 $1^{\mathrm{a}}$ ed. 2001]: Os petróglifos e o seu contexto. Instituto de Estudios Vigueses. Vigo.

Filgueiras Rey, A. I. y Rodríguez Fernández, T. 1994: "Túmulos y petroglifos. La construcción de un espacio funerario. Aproximación a sus implicaciones simbólicas. Estudio en la Galicia centro-oriental: Samos y Sarria". Espacio, Tiempo y Forma, Serie I, Prehistoria y Arqueología 7: 211-253.

Fischer, P. 1992: "First experiments in viewshed uncertainty: simulating fuzzy viewsheds". Photogrammetric engineering and remote sensing 58: 345-352.

Fischer, P. F. 1993: "Algorithm and implementation uncertainity in viewshed analysis". International Journal of Geographical Information Science 7: 331-347. https://doi.org/10.1080/02693799308901965

Fischer, P. F.; Farrelly, C.; Maddocks, A. y Ruggles, C. L. N. 1997: "Spatial Analysis of visible areas from the Bronze Age cairns of Mull”. Journal of Archaeological Science 24: 581-592. https://doi.org/10.1006/jasc.1996.0142

Fleming, A. 1973: "Tombs for the living". Man 8 (2): 177-193. https://doi.org/10.2307/2800845

García, L.; Metcalfe-Wood, S.; Rivera, T. y Wheatley, D. W. 2006: “Análisis de pautas de visibilidad en la distribución de monumentos megalíticos de Sierra Morena Occidental”. En I. Grau Mira (ed.): La aplicación de los SIG en la arqueología del paisaje. Universidad de Alicante. Alicante: 181-200.

García Sanjuán, L. 2000: “Grandes piedras, paisajes sagrados". Boletín del Instituto Andaluz de Patrimonio Histórico 31: 171-178.

García Sanjuán, L. 2008: "Muerte, tiempo, memoria. Los megalitos como memoriales culturales". PH, Boletín Andaluz del Patrimonio Histórico 67: 34-45. 
Herzog, I. 2013a: "The potential and limits of optimal path analysis". In A. Bevan y M. Lake (eds.): Computational approaches to archaeological spaces. Left Coast Press. Walnut Creek: 179-211.

Herzog, I. 2013b: "Theory and practice of cost functions". En F. Contreras Cortés, M. Farjas y F. J. Melero (eds.): Proceedings of the 38th Annual Conference on Computer Applications and Quantitative Methods in Archaeology. Archaeopress. Oxford: 375-382.

Higuchi, T. 1983: Visual and spatial structure of landscapes. MIT. Massachusetts.

Holmgren, P. 1994: "Multiple flow direction algorithms for runoff modelling in grid based elevation models: an empirical evaluation". Hydrological Processes 8 (4): 327-334. https://doi.org/10.1002/hyp.3360080405

Infante Roura, F.; Vaquero Lastres, J. y Criado Boado, F. 1992: "Vacas, caballos, abrigos y túmulos: definición de una geografía del movimiento para el estudio arqueológico". Cuadernos de Estudios Gallegos 40 (105): 22-39. https://doi.org/10.3989/ceg.1992.v40.i105.297

Kvamme, K. L. 1988: "Development and testing of quantitative models". En W. J. Judge y L. Sebastian (eds.): Quantifying the present and predicting the past: theory, method and application of archaeological predictive modeling. US Bureau of Land Management. Washington: 325-427.

Lake, M. y Woodman, P. 2000: "Viewshed analysis of site location on Islay". En S. J. Mithen (ed.): Hunter-gatherer landscape archaeology: the Southern Hebrides Mesolithic Project, 1988-98. The McDonald Institute for Archaeological Research. Cambridge: 497-503.

Lake, M. y Woodman, P. 2003: "Visibility studies in archaeology: a review and case study". Environment and planning B: planning and design 30: 689-707. https://doi.org/10.1068/b29122

Llobera, M. 2000: "Understanding movement: a pilot model towards the sociology of movement". En G. Lock (ed.): Beyond the map: archaeology and spatial technologies. IOS Press. Washington DC: 66-84.

Llobera, M. 2003: "Extending GIS-based visual analysis: The concept of 'visualscapes"'. International Journal of Geographical Information Science 17: 25-48. https://doi.org/10.1080/713811741

Llobera, M. 2006a: "What you see is what you get? Genesis and hierarchy in visualscapes". En T. L. Evans y P. Daly (eds.): Digital archaeology. Bridging method and theory. Routledge. Oxford: 132-151.

Llobera, M. 2006b: "Arqueología del Paisaje en el siglo XXI. Reflexiones sobre el uso de los SIG y modelos matemáticos". En I. Grau Mira (ed.): La aplicación de los SIG en la arqueología del paisaje. Servicio de Publicaciones Universidad de Alicante. Alicante: 109-124.

Llobera, M. 2007: "Reconstructing visual landscapes". World Archaeology 39 (1): 51-69. https://doi.org/10.1080/00438240601136496

Llobera, M. 2015: "Working the digital: some thoughts from landscape archaeology". En R. Chapman y A. Wylie (eds.): Material evidence: learning from archaeological practice. Routledge. Abingdon: 173-188.

Llobera, M.; Wheatley, D.; Steele, J.; Cox, S. y Parchment, O. 2010: "Calculating the inherent visual structure of a landscape ("total viewshed") using high-throughput computing". En F. Nicolucci y S. Hermon (eds.): Beyond the artifact. Digital interpretation of the past. Proceedings of CAA2004 (Prato 2004). Archaeolingua. Budapest: $146-151$

López Cuevillas, F. 1925: "As mámoas do Concello de Lobeira". Boletín de la Real Academia Gallega XV: 25-39.

López Cuevillas, F. 1933: "Prehistoria de Melide". En R. E. Otero Pedrayo (ed.): Terra de Melide. Seminario de Estudos Galegos. Santiago de Compostela.

López Plaza, J. y Salvador Mateos, R. 2002: "Megalitismo y vías naturales de comunicación en el SO Salmantino". In V. M. Oliveira Jorge (ed.): 3er Congreso de Arqueología Peninsular (Vila Real 1999). Porto: 271-87.

López-Romero, E. 2007: "Factores visuales de localización de los monumentos megalíticos de la cuenca del Sever (Portugal-España)". Trabajos de Prehistoria 64 (2): 73-93. https://doi.org/10.3989/tp.2007.v64.i2.110

Maciñeira, F. 1929: "Notable grupo de círculos líticos y túmulos dolménicos de la cuenca superior del Eume". Arquivos do Seminario de Estudos Galegos 2: 197-218.

Maciñeira, F. 1935: "La distribución de las estaciones prehistóricas ortegaleses y sus características". Boletín de la Real academia Galega 259: $169-181$
Maciñeira, F. 1943-1944: “Túmulos prehistóricos. Inventario descriptivo de los 286 túmulos prehistóricos hasta ahora descubiertos en la avanzada comarca del cabo Ortegal". Boletín de la Real Academia Galega 23-24: 15-34.

Manly, B. F. J. 1991: Randomization and Monte Carlo methods in Biology. Chapman and Hall. London.

Martinón-Torres, M. 2001: Os monumentos megalíticos despois do megalitismo. Arqueoloxía e historia dos megalitos a través das fontes escritas (S. VI-S. XIX). Concello de Valga. Valga.

Mlekuz, D. 2014: "Exploring the topography of movement". En S. Polla y P. Verhagen (eds.): Computational approaches to the study of movement in Archaeology: theory, practice and interpretation of factors and effects of long term landscape formation and transformation. De Gruyter. Berlin: 5-21.

Murrieta Flores, P. 2010: "Travelling in a prehistoric landscape: exploring the influences that shaped human movement". En J. Frischer, J. Webb Crawford y D. Koller (eds.): Making history interactive. Computer applications and quantitative methods in Archaeology (CAA). Proceedings of the $37^{\text {th }}$ International Conference (Williamsburg, Virginia, United States of America 2009). British Archaeological Reports, International Series 2079, Archaeopress. Oxford: 258-276.

Murrieta Flores, P. 2012: "Understanding human movement through spatial technologies: the role of natural areas of transit in the Late Prehistory of South-western Iberia". Trabajos de Prehistoria, 69 (1): 103-122. https://doi.org/10.3989/tp.2012.12082

Murrieta Flores, P. 2014: "Developing computational approaches for the study of movement: the impact of visibility in terrestrial navigation during Iberian Late Prehistory". En S. Polla y P. Verhagen (eds.): Computational approaches to the study of movement in Archaeology. Theory, practice and interpretation of factors and effects of long term landscape formation and transformation. De Gruyter. Berlin: 99-132.

Murrieta Flores, P.; García Sanjuán, L. y Wheatley, D. 2014: "Modelando la movilidad y el movimiento a través de los SIG. Estudios de caso en la Prehistoria Reciente de Sierra Morena Occidental”. En E. García Alfonso (ed.): Movilidad, contacto y cambio. II Congreso de Prehistoria de Andalucia (Antequera 2012): 77-92. Sevilla.

Murrieta Flores, P.; Wheatley, D. y García Sanjuán, L. 2011a: "Antes de los mapas: navegación y orientación terrestre en la Prehistoria Reciente Ibérica". PH: Boletín del Instituto Andaluz del Patrimonio Histórico 19 (77): 85-88.

Murrieta Flores, P. A.; Wheatley, D. y García Sanjuán, L. 2011b: "Movilidad, trashumancia y paisaje prehistórico: Estudio del paisaje arqueológico de Almadén de la Plata, Andalucía a través de un SIG". En V. Mayoral Herrera y S. Celestino Pérez (eds.): Tecnologías de información geográfica y análisis arqueológico del territorio. Actas del V Simposio Internacional de Arqueología de Mérida (Mérida 2007). Anejos del Archivo Español de Arqueología LIX. Instituto de Arqueología-Mérida, CSIC-Junta de Extremadura. Mérida: 411-423.

Ogburn, D. E. 2006: "Assessing the level of visibility of cultural objects in past landscapes". Journal of Archaeological Science 33 (3): 405-413. https://doi.org/10.1016/j.jas.2005.08.005

Orengo, H. A. y Livarda, A. 2016: "The seeds of commerce: a network analysis-based approach to the Romano-British transport system". Journal of Archaeological Science 66: 21-35. https://doi.org/10.1016/j.jas.2015.12.003

Paliou, E. 2013: "Reconsidering the concept of visualscapes: recent advances in three-dimensional visibility analysis". En A. Bevan y M. Lake (eds.): Computational approaches to archaeological spaces. Left Coast Press. Walnut Creek, California: 243-263.

Parcero Oubiña, C. y Fábrega Álvarez, C. 2006: "Diseño metodológico para el análisis locacional de asentamientos a través de un SIG de base ráster". En I. Grau Mira (ed.): La aplicación de los SIG en la Arqueología del Paisaje. Servicio de Publicaciones Universidad de Alicante. Alicante: 69-91.

Rášová, A. 2014: "Fuzzy viewshed, probable viewshed, and their use in the analysis of prehistoric monuments placement in Western Slovakia”. En J. Huerta, S. Schade y C. Granel (eds.): Connecting a digital Europe through location and place. Proceedings of the AGILE'2014 International Conference on Geographic Information Science. Springer. Castellón. http://repositori.uji.es/xmlui/handle/10234/99490 (consulta 12-11-2017). 
Rodríguez Casal, A. A. 1998: "La necrópolis megalítica del Monte de Santa Mariña". Gallaecia 17: 121-135.

Rodríguez Casal, A. A.; Gómez Nistal, C. y Romaní Fariña, E. 1998: "El fenómeno tumular y megalítico en las tierras de Sarria-O Incio (Lugo)". Gallaecia 17: 69-105.

Rodríguez Rellán, C. y Fábregas Valcarce, R. 2015: “Arte rupestre galaica: unha achega dende a estatística espacial e os SIX". Sémata, Ciencias Sociais e Humanidades 27: 323-348.

Vaquero Lastres, J. 1990: "Ríos y tumbas. Sobre el emplazamiento de túmulos en el NW peninsular". Trabalhos de Antropologia e Etnologia XXX (1-4): 151-173.

Vaquero Lastres, J. 1991-1992: "Del análisis del emplazamiento al estudio de la distribución de túmulos en el NW". Brigantium 7: 151-176.

Vaquero Lastres, J. 1993-1994: “Galiñeiro, paso de lobos, novíos y héroes. Sobre el emplazamiento de los túmulos del Noroeste". Cuadernos de Estudios Gallegos XLI (106): 11-39. https://doi.org/10.3989/ceg.1994.v41.i106.254

Verhagen, P. 2013: "On the road to nowhere? Least cost paths, accessibility and the predictive modelling perspective". En F. Contreras Cortés, M. Farjas y F. J. Melero (eds.): Proceedings of the $38^{\text {th }}$ Annual Conference on Computer Applications and Quantitative Methods in Archaeology. Archaeopress. Oxford: 384-390.

Wheatley, D. W.; García Sanjuán, L.; Murrieta Flores, P. y Márquez Pérez, J. 2010: "Approaching the landscape dimension of the megalithic phenomenon in Southern Spain". Oxford Journal of Archaeology 29 (4): 387-405. https://doi.org/10.1111/j.1468-0092.2010.00354.x

Wheatley, D. y Gillings, M. 2000: "Vision, perception and GIS: developing enriched approaches to the study of archaeological visibility". In G. Lock (ed.): Beyond the map: archaeology and spatial technologies. IOS Press. Amsterdam: 1-27.

Wheatley, D. y Gillings, M. 2002: Spatial technology and archaeology: the archaeological applications of GIS. Taylor and Francis. London.
White, D. A. y Barber, S. B. 2012: "Geospatial modelling of pedestrian transportation networks: a case study from precolumbian Oaxaca". Journal of Archaeological Science 39: 2684-2696. https://doi.org/10.1016/j.jas.2012.04.017

Whitley, T. G. y Hicks, L. M. 2003: "A geographic information systems approach to understanding potential prehistoric and historic travel corridors". Southeast Archaeology 22: 77-91.

Yubero Gómez, M.; Rubio Campillo, X.; López Cachero, F. J. y Esteve Gràcia, X. 2015: "Mapping changes in late prehistoric landscapes: a case study in the north-eastern Iberian Peninsula". Journal of Anthropological Archaeology 40: 123-134. https://doi.org/10.1016/j.jaa.2015.07.002

\section{ARCHIVOS COMPLEMENTARIOS}

En la versión en línea pueden encontrarse adjuntos cuatro archivos en formato Excel, correspondientes a los monumentos megalíticos (coordenadas UTM, ED50) y el estudio del tamaño de la cuenca visual ("vsize"), tanto para los monumentos megalíticos como para el conjunto de las simulaciones aleatorias definidas en el trabajo.

AC1: Monumentos megalíticos.

AC2: Tamaño de la cuenca visual de los túmulos megalíticos.

AC3: Tamaño de la cuenca visual de la muestra aleatoria sin condicionar.

AC4: Tamaño de la cuenca visual de la muestra aleatoria condicionada. 